\title{
Trifluoromethyl Vinyl Sulfide: A Building Block for the Synthesis of $\mathrm{CF}_{3} \mathrm{~S}$-Containing Isoxazolidines
}

\author{
Alejandra Riesco-Domínguez, ${ }^{\dagger}$ Jeroen van de Wiel, $^{\dagger}$ Trevor A. Hamlin, $^{\ddagger}{ }^{\circ}$ Bas van Beek, ${ }^{\ddagger}$
} Stephen D. Lindell, ${ }^{\S}$ Daniel Blanco-Ania, ${ }^{\dagger}$ F. Matthias Bickelhaupt, ${ }^{*},+\neq(0)$ and Floris P. J. T. Rutjes ${ }^{*}+{ }^{\dagger}$

${ }^{\dagger}$ Radboud University, Institute for Molecules and Materials, Heyendaalseweg 135, 6525 AJ Nijmegen, The Netherlands

${ }^{\ddagger}$ Department of Theoretical Chemistry and Amsterdam Center for Multiscale Modeling (ACMM), Vrije Universiteit Amsterdam, De Boelelaan 1083, $1081 \mathrm{HV}$ Amsterdam, The Netherlands

${ }^{\S}$ Bayer CropScience AG, Industriepark Höchst, G836, 65926 Frankfurt am Main, Germany

Supporting Information

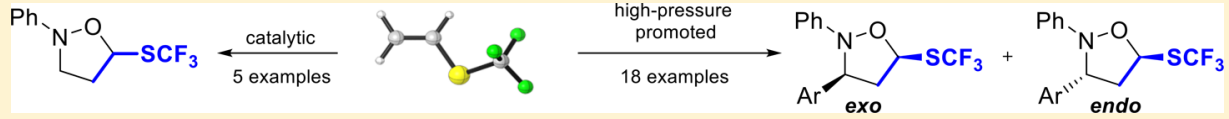

ABSTRACT: Trifluoromethyl vinyl sulfide, a potential building block for pharmaceutically and agrochemically relevant products, is prepared and used for the first time in high-pressure-mediated 1,3-dipolar cycloaddition reactions with nitrones to synthesize (trifluoromethyl)sulfanyl isoxazolidines.

\section{INTRODUCTION}

The (trifluoromethyl)sulfanyl group $\left(\mathrm{SCF}_{3}\right)$ represents a privileged substituent in agrochemicals and pharmaceuticals because of the strong electron-withdrawing effect and the large Hansch lipophilicity parameter $(\pi=1.44) .{ }^{1}$ Cefazaflur, $^{2}$ a firstgeneration cephalosporin antibiotic, toltrazuril, ${ }^{3}$ an antiprotozoal agent, and a losartan analogue, ${ }^{4}$ developed as a potential hypotensive agent, are prominent examples of biologically active compounds bearing the $\mathrm{SCF}_{3}$ group (Figure 1).

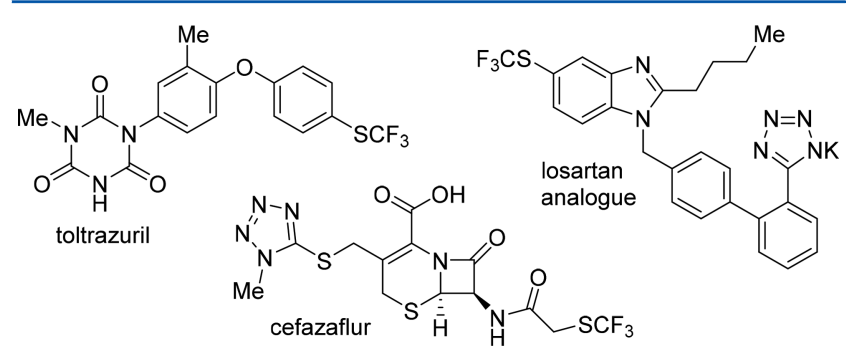

Figure 1. Bioactive heterocycles containing the $\mathrm{SCF}_{3}$ group.

Hence, $\mathrm{CF}_{3} \mathrm{~S}$-containing compounds are considered appealing targets in the agrochemical and pharmaceutical fields, and consequently, modern research has focused on efficient (trifluoromethyl)sulfanylation methods. The main strategies that have been developed to synthesize $\mathrm{CF}_{3} \mathrm{~S}$-containing compounds have focused on direct $\mathrm{C}-\mathrm{S}$ bond formation ${ }^{5}$ and trifluoromethylation of sulfur-containing compounds. ${ }^{6}$ However, incorporation via simple $\mathrm{CF}_{3} \mathrm{~S}$-containing building blocks is still unprecedented so that the use of a $\mathrm{CF}_{3} \mathrm{~S}$-containing reagent could be an alternative strategy to construct $\mathrm{CF}_{3} \mathrm{~S}$ substituted heterocycles. More specifically, trifluoromethyl vinyl sulfide (2) could be an attractive building block to provide straightforward access to a wide variety of hetero- and carbocycles via cycloaddition reactions. Previously reported examples of cycloaddition reactions of alkene $\mathbf{2}$ are rather scarce: there is a single example of a Diels-Alder reaction with 2,3dimethylbutadiene to yield the corresponding $\mathrm{CF}_{3} \mathrm{~S}$-cyclohexene, 7 and two cyclopropanation examples with organomercury reagents to yield $\mathrm{CF}_{3} \mathrm{~S}$-substituted cyclopropanes.

The potential of this unexplored chemistry and the inherent biological attractiveness of these molecules motivated us to further study the reactivity of alkene $\mathbf{2}$ in cycloaddition reactions. Thus, we herewith present the study of the first 1,3dipolar cycloaddition reactions of 2 with several nitrones ${ }^{9}$ to synthesize a novel group of isoxazolidines (Scheme 1).

Scheme 1. Synthesis of $\mathrm{CF}_{3} \mathrm{~S}$-Containing Isoxazolidines

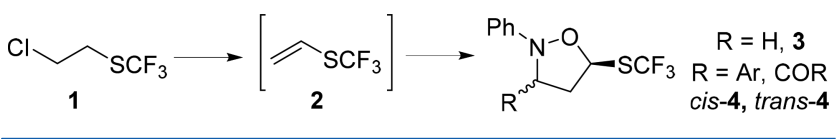

\section{RESULTS AND DISCUSSION}

We commenced our investigations by forming alkene 2 in situ. Because of its high volatility, we first performed the elimination reactions in deuterated solvents in order to instantly measure conversions from commercially available chloro alkane $\mathbf{1}$ into alkene 2, with no need for further treatment or isolation of the alkene. We tested several bases $\left(\mathrm{Et}_{3} \mathrm{~N}, \mathrm{DBU}, \mathrm{KO}^{t} \mathrm{Bu}, \mathrm{KOH}\right.$ and KOTMS) in the presence of various deuterated solvents $\left(\mathrm{CD}_{2} \mathrm{Cl}_{2}\right.$, THF- $d_{8}, \mathrm{CD}_{3} \mathrm{OD}$ and DMF- $d_{7}$; see the Supporting Information (SI) for experimental details) to eventually choose

Received: October 17, 2017

Published: January 10, 2018 
a solution of $\mathrm{KO}^{t} \mathrm{Bu}$ in THF- $d_{8}$ at $21{ }^{\circ} \mathrm{C}$ for $90 \mathrm{~min}$ as the final method for the in situ synthesis of alkene 2 with full conversion.

Initially, we focused on the synthesis of 2,5-disubstituted isoxazolidines 3 using $\mathrm{N}$-substituted hydroxylamines $\mathbf{5}$ and paraformaldehyde as precursors for the in situ synthesis of the dipoles (Table 1). The nitrone intermediates were used without

Table 1. Synthesis of Isoxazolidines $3 a-e$

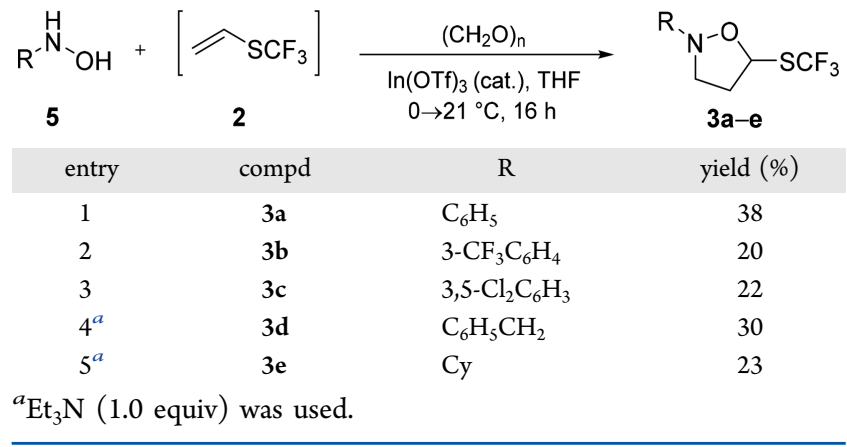

isolation in a one-pot process with a preformed solution of alkene 2 ( 3 equiv) and $\operatorname{In}(\mathrm{OTf})_{3}$ (0.06 equiv). In this manner, we obtained isoxazolidine $3 a$ in $22 \%$ yield after column chromatography. Increasing the concentration of 2 (6 equiv) resulted in a yield of $38 \%$ (entry 1 ). Isoxazolidines $3 \mathbf{b}-\mathbf{e}$ were synthesized in yields between 20 and $30 \%$ under the same reaction conditions with different substrates (entries 2-5). However, yields were rather low after column chromatography in all cases (see the SI for experimental details).

With these results in hand and because of the low yields in the synthesis of $3 \mathbf{a}-\mathbf{e}$, we changed our strategy to the synthesis of 2,3,5-trisubstituted isoxazolidines 4, exhibiting a second (hetero)aromatic group at the 3-position and introducing consequently a second chiral center in the molecule (Scheme 2, Table 2).

Scheme 2. Formation of Compounds 4, 7, and 8

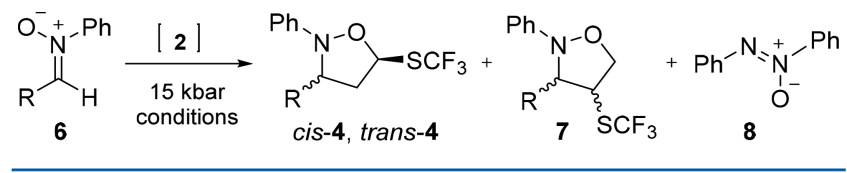

We first performed the reaction with nitrone $\mathbf{6 a}(\mathrm{R}=\mathrm{H})$, a catalytic amount of $\operatorname{In}(\mathrm{OTf})_{3}$, and alkene 2 at $21^{\circ} \mathrm{C}$ in THF, but no conversion into the product was observed. When the same conditions were applied at $80^{\circ} \mathrm{C}$ with nitrone $6 \mathrm{c}(\mathrm{R}=\mathrm{Me})$, less than $7 \%$ conversion into the final product was observed by ${ }^{1} \mathrm{H}$ NMR, presumably because of the low reactivity of this alkene and perhaps due to its volatility. Therefore, we decided to perform these reactions under high-pressure conditions (Table 2 ), which is an established and powerful tool to increase the rate of cycloaddition reactions. ${ }^{10}$ Thus, when 1.3 equiv of 2 at $21^{\circ} \mathrm{C}$ under a $15 \mathrm{kbar}$ pressure was used (entry 1) we gratifyingly observed an $83 \%$ conversion into isoxazolidines $4 \mathrm{c}$ and $7 \mathrm{c}$ (regioisomeric ratio $4 \mathrm{c} / 7 \mathrm{c}$ 13:1, diastereomeric ratio $[\mathrm{dr}]$ cis$4 \mathrm{c} /$ trans-4c $7: 2$ ). Nitrone $6 \mathrm{~b}$ gave only $56 \%$ conversion under the same reaction conditions (entry 4), with similar regio- and diastereoisomeric ratios. When increasing the concentration of alkene 2, 93 and $75 \%$ conversions into compounds $\mathbf{4 c}$ and $\mathbf{4 b}$ were obtained, respectively (entries 2 and 5). Finally, an increase of the temperature to $50{ }^{\circ} \mathrm{C}$ gave nearly full conversion into the desired cis- and trans-products $4 c$ and $4 b$ (entries 3 and 6).

Azoxybenzene $^{11} \mathbf{8}$ was formed in all reactions as a side product (Scheme 2) and could not be separated in any case from trans-4 by column chromatography. Thus, we used galvinoxyl to prevent the formation of compound 8 . $^{12} \mathrm{We}$ were pleased to see that when using 3 equiv of alkene 2 at $50{ }^{\circ} \mathrm{C}$, in the presence of 1-3 mol \% of galvinoxyl as radical scavenger (entries 7 and 8), not only full conversion into the desired product $\mathbf{4 b}$ was obtained, but also the formation of the azoxy compound $\mathbf{8}$ was fully suppressed.

Furthermore, our reactions gave the exo-products as the major diastereoisomers (the cis-isomers), showing a 3:1 diastereomeric ratio (entries 7 and 8). Having these results in hand, the scope of nitrones in this 1,3-dipolar cycloaddition was examined by employing the conditions shown in entry 7 . We first synthesized a total of 18 nitrones $(6 a-r)$ with phenyl (containing both electron-donating and electron-withdrawing groups at the 2-, 3-, and 4- positions), heterocyclic and carbamoyl substituents in excellent yields (see the SI). We then studied the scope of the 1,3-dipolar cycloaddition reactions between alkene $\mathbf{2}$ and nitrones $\mathbf{6 a}-\mathbf{r}$ (Table 3).

As shown in Table 3, high and excellent yields (total yields) were observed for compounds $4 \mathbf{a}-\mathbf{c}, \mathbf{4} \mathbf{f}-\mathbf{i}, 4 \mathbf{l}$, and $4 \mathbf{n}$. In addition, in most of the cases the major product (cis-4) was separated from the other isomers by column chromatography in good yields (cis-4a-c, cis-4f-j, cis-4m, and cis-4q). Unfortunately, in a few cases the isomers 4 were obtained as a mixture that could not be separated (4d,e, $\mathbf{4 k}, \mathbf{l}$, and $\mathbf{4 n}$ ). For compounds 4o and $4 \mathrm{p}$, the trans-4 isomer and the corresponding regioisomers 7 were present as a mixture. ${ }^{13}$ The assignment of the cis- and trans-isomers was performed by 2D NMR studies (NOESY).

The diastereoselectivity was lower for compounds 40 and $\mathbf{4 p}$, which contained an indole and a pyrrole substituent, respectively. In these cases, a mixture of cis- and transdiastereoisomers was obtained approximately in a 3:2 ratio. In

Table 2. Optimization Process for the 1,3-Dipolar Cycloaddition Reaction of Nitrones 6 with Alkene 2

\begin{tabular}{|c|c|c|c|c|c|c|c|c|}
\hline entry & nitrone & $\mathrm{R}$ & 2 (equiv) & $T\left({ }^{\circ} \mathrm{C}\right)$ & scavenger & cis-4/trans-4 & $8^{a}(\%)$ & $\operatorname{conv}^{a}(\text { yield })^{b}(\%)$ \\
\hline 1 & $6 c$ & 4- $\mathrm{MeC}_{6} \mathrm{H}_{4}$ & 1.3 & 21 & & $76: 22$ & 5 & $83(41)^{c}$ \\
\hline 2 & $6 c$ & 4- $\mathrm{MeC}_{6} \mathrm{H}_{4}$ & 3 & 21 & & $79: 21$ & 22 & $93(-)$ \\
\hline 3 & $6 c$ & 4- $\mathrm{MeC}_{6} \mathrm{H}_{4}$ & 3 & 50 & & $76: 24$ & 6 & $96(85)^{c}$ \\
\hline 4 & $6 b$ & 4- $\mathrm{FC}_{6} \mathrm{H}_{4}$ & 1.3 & 21 & & $79: 21$ & 7 & $56(35)^{c}$ \\
\hline 5 & $6 b$ & 4- $\mathrm{FC}_{6} \mathrm{H}_{4}$ & 3 & 21 & & $79: 21$ & 38 & $75(-)$ \\
\hline 6 & $6 b$ & $4-\mathrm{FC}_{6} \mathrm{H}_{4}$ & 3 & 50 & & $77: 23$ & 17 & $91(79)^{c}$ \\
\hline 7 & $6 b$ & $4-\mathrm{FC}_{6} \mathrm{H}_{4}$ & 3 & 50 & galvinoxyl (3\%) & $76: 24$ & 0 & $100(78)$ \\
\hline 8 & $6 b$ & 4- $\mathrm{FC}_{6} \mathrm{H}_{4}$ & 3 & 50 & galvinoxyl (1\%) & $76: 24$ & 1 & $100(-)$ \\
\hline
\end{tabular}

${ }^{a}$ Calculated by integration of the ${ }^{1} \mathrm{H}$ NMR signals of the crude mixtures. ${ }^{b}$ Isolated after column chromatography. ${ }^{c}$ trans-Isomer contaminated with 7. 
Table 3. Scope of the High-Pressure-Promoted 1,3-Dipolar Cycloaddition Reaction

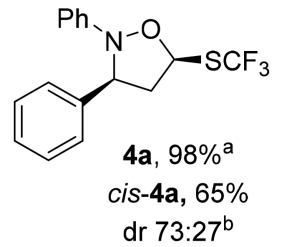<smiles>COc1ccc(C2CC(C(F)(F)F)ON2c2ccccc2)cc1</smiles>

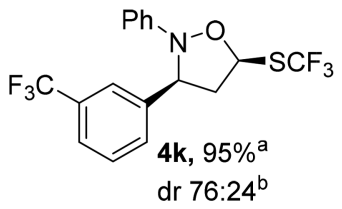
dr $76: 24^{b}$
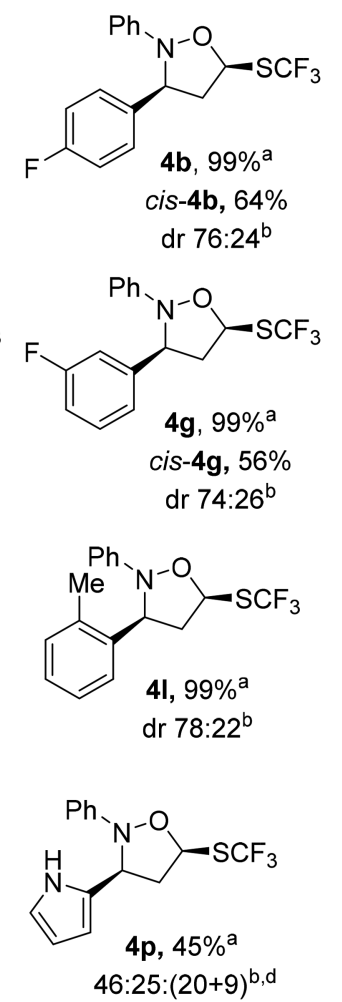<smiles>Cc1ccc(C2CC(C(F)(F)F)ON2c2ccccc2)cc1</smiles><smiles>C#[Ge]C1C[C@@H](c2ccc(C(F)(F)F)cc2)N(c2ccccc2)O1</smiles><smiles>FC(F)(F)c1ccc([C@H]2CC(C(F)(F)F)ON2c2ccccc2)cc1</smiles><smiles>Cc1cccc(C2CC(C(F)(F)F)ON2c2ccccc2)c1</smiles><smiles>COC(=O)c1cccc(C2CC(C(F)(F)F)ON2c2ccccc2)c1</smiles><smiles>COc1cccc(C2CC(C(F)(F)F)ON2c2ccccc2)c1</smiles>
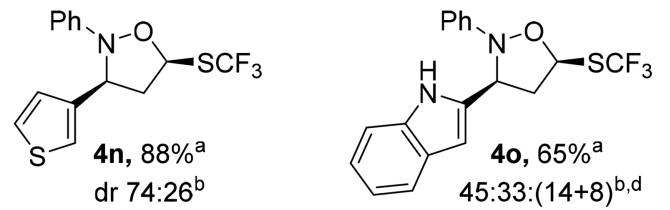

-4m, 43\%

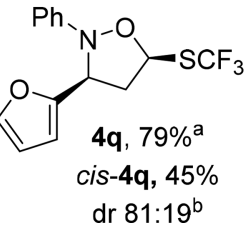

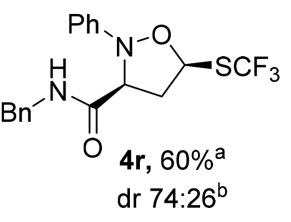

${ }^{a}$ Combined yield. ${ }^{b}$ Calculated by ${ }^{1} \mathrm{H}$ NMR of the crude. ${ }^{c}$ Galvinoxyl was not used in the reaction. ${ }^{d}$ cis-4o,p/trans-4o,p/(mixture of isomers 7$)$.

addition, for compounds 40 and $4 \mathrm{p}$, a $7: 3$ ratio of a mixture cis-7 and trans-7 from the regioisomer 7 was observed. For these two compounds (4o and $4 \mathrm{p}$ ), the total yields were significantly lower because of incomplete conversion into the products and purification problems. Finally, when using a nonaromatic nitrone, the final benzyl amide isoxazolidine $4 \mathbf{r}$ was synthesized in $60 \%$ yield.

To evaluate the apparent regioselectivity of the cycloaddition, high-level DFT calculations were carried out at the BP86/ $\mathrm{TZ}_{2} \mathrm{P}^{14}$ level, using the ADF program. ${ }^{15}$ Tetrahydrofuran was simulated using the COSMO solvation model. ${ }^{16}$ Thermochemical corrections were computed using the temperature and pressure under which the experiments were performed (see the SI for computational details).

We focused on understanding the regioselectivity of the cycloaddition between a simple model nitrone 9 with alkene 2 (Scheme 3). This reaction proceeds in a concerted and

Scheme 3. Formation of Compounds 10 and 11

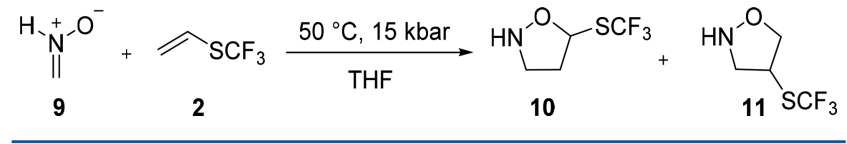

asynchronous manner with a Gibbs free energy barrier of 21.3 and $24.0 \mathrm{kcal} \mathrm{mol}^{-1}$ for $\mathbf{1 0}$ and $\mathbf{1 1}$, respectively (Table 4). The $\Delta \Delta G^{\ddagger}$ of $2.7 \mathrm{kcal} \mathrm{mol}^{-1}$ facilitates a high degree of regioselectivity, resulting in a calculated product ratio of $98: 2$ for 10/11. Furthermore, the experimentally observed regioisomer 10 is more stable than $11\left(\Delta \Delta G_{\mathrm{rxn}}=3.9 \mathrm{kcal}\right.$ $\left.\mathrm{mol}^{-1}\right)$. The calculated product ratio for the model nitrone cycloaddition is in line with experimentally observed regioselectivity of reactions involving the majority of the nitrones 6 .
Table 4. Computed Activation Barriers, Reaction Energies $\left(\mathrm{kcal} \mathrm{mol}^{-1}\right)$, and Product Distribution Computed at the COSMO(THF)-BP86/TZ2P Level of Theory

$\begin{array}{cccc}\text { compd } & \Delta E^{\ddagger}\left(\Delta G^{\ddagger}\right) & \Delta E_{\mathrm{rxn}}\left(\Delta G_{\mathrm{rxn}}\right) & \text { product ratio }^{a} \\ 10 & 12.8(21.3) & -17.6(-6.1) & 98 \\ 11 & 15.0(24.0) & -14.3(-2.2) & 2\end{array}$

${ }^{a}$ Calculated at $50{ }^{\circ} \mathrm{C}$ and $15 \mathrm{kbar}$ based on the $\Delta \Delta G^{\ddagger}$ between 10 and 11 (see the SI for details).

Insight into why regioisomer $\mathbf{1 0}$ is favored over the other, $\mathbf{1 1}$ is provided by the activation strain model (ASM) ${ }^{17}$ (also known as the distortion-interaction model). ${ }^{18}$ In this framework, the potential energy surface $\Delta E(\zeta)$ is decomposed along the reaction coordinate $\zeta$ into the strain $\Delta E_{\text {strain }}(\zeta)$ associated with deforming the individual reactants plus the actual interaction $\Delta E_{\text {int }}(\zeta)$ between the deformed reactants.

$$
\Delta E^{\ddagger}(\zeta)=\Delta E_{\text {strain }}+\Delta E_{\text {int }}(\zeta)
$$

The $\Delta E_{\text {int }}(\zeta)$ between the reactants is further analyzed by an energy decomposition analysis (EDA) in the conceptual framework provided by the Kohn-Sham molecular orbital (KS-MO) model $^{19}$ and is decomposed into three physically meaningful terms:

$$
\Delta E_{\text {int }}(\zeta)=\Delta V_{\text {elstat }}(\zeta)+\Delta E_{\text {Pauli }}(\zeta)+\Delta E_{\text {oi }}(\zeta)
$$

The $\Delta V_{\text {elstat }}(\zeta)$ term corresponds to the classical electrostatic interaction between unperturbed charge distributions, $\Delta E_{\text {Pauli }}(\zeta)$ is responsible for any steric repulsion, and the $\Delta E_{\mathrm{oi}}(\zeta)$ accounts for charge transfer (HOMO-LUMO interactions) and polarization.

Applying the ASM along the reaction coordinate defined by the bending of the 1,3-dipole, it is revealed that reactivity 
differences in the reaction between 9 and 2 leading to 10 (blue curve) and 11 (red curve) are a result of $\Delta E_{\text {int }}$ (Figure 2a).
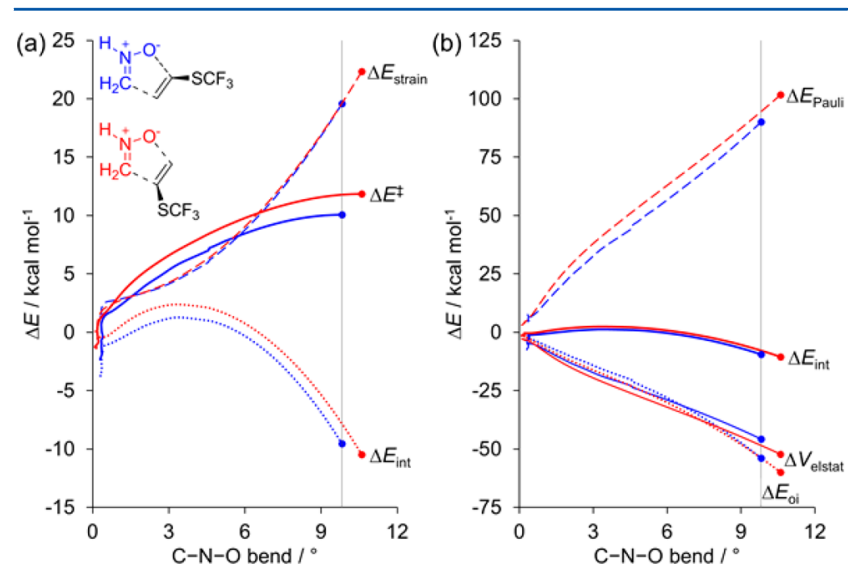

Figure 2. (a) Activation strain and (b) energy decomposition analyses of the cycloaddition reactions of nitrone 9 with alkene 2 up to their respective TS (indicated by the dot) computed at the BP86/TZ2P level.

$\Delta E_{\text {strain }}$ remains nearly constant for both reactions along the reaction coordinate. Next, the EDA terms were analyzed, and the $\Delta E_{\text {Pauli }}$ dominates and is chiefly responsible for the difference in the interaction energies (Figure $2 b$ ). The more favorable $\Delta V_{\text {elstat }}$ and $\Delta E_{\mathrm{oi}}$ curves associated with the reaction leading to $\mathbf{1 1}$ are unable to overcome for increased steric repulsion associated with this approach. These results highlight the fact that the reaction results from the least hindered approach, leaving the $\mathrm{SCF}_{3}$ group far away from the $\mathrm{CH}_{2}$ group. See the SI for an ASA and EDA analysis on $6 \mathrm{~m}$ and $6 \mathrm{p}$.

In summary, we have developed the synthesis of a new class of 5-[(trifluoromethyl)sulfanyl]isoxazolidines. The reactions utilized for their synthesis were high-pressure-promoted 1,3dipolar cycloaddition reactions between trifluoromethyl vinyl sulfide 2 and various easily synthesized nitrones. The results of our DFT computations are in harmony with experimental results and were leveraged to show that the high regioselectivity of these cycloaddition reactions originates from minimizing steric repulsion.

\section{EXPERIMENTAL SECTION}

General Information. Reagents were obtained from commercial suppliers and were used without purification. Standard syringe techniques were applied for the transfer of dry solvents and air- or moisture-sensitive reagents. Tetrahydrofuran was used as a solvent after distillation. Potassium tert-butoxide was used as a $1.0 \mathrm{M}$ solution in tetrahydrofuran. The high-pressure experiments were run in a highpressure apparatus equipped with a one-wall-piston cylinder for pressures up to $15 \mathrm{kbar}(1.5 \mathrm{GPa})$. Reactions were performed in 1$1.5 \mathrm{~mL}$ PTFE ampules closed by screwed stainless steel stoppers. These ampules were inserted into the high-pressure vessel filled with 80-100 petroleum ether as transmission medium. Reactions were followed, and $R_{f}$ values were obtained, using thin layer chromatography (TLC) on silica gel-coated plates (Merck 60 F254) with the indicated solvent mixture. Detection was performed with UV light, and/or by charring at ca. $150{ }^{\circ} \mathrm{C}$ after dipping into a solution of $\mathrm{KMnO}_{4}$. Infrared spectra were recorded on an IR-ATR Bruker TENSOR 27 spectrometer. Highresolution mass spectra were recorded on a JEOL AccuTOF (ESI) or a MAT900 (EI, CI, and ESI) GC-TOF. NMR spectra were recorded at $298 \mathrm{~K}$ on a Varian Inova 400 (400 MHz), Bruker Avance III $400 \mathrm{MHz}$ or Bruker Avance III $500 \mathrm{MHz}$ spectrometer in the solvent indicated. Chemical shifts are given in parts per million (ppm) with respect to tetramethylsilane $(0.00 \mathrm{ppm})$ as internal standard for ${ }^{1} \mathrm{H}$ NMR; and $\mathrm{CDCl}_{3}$ (77.16 ppm) as internal standard for ${ }^{13} \mathrm{C}$ NMR. Coupling constants are reported as $J$ values in hertz $(\mathrm{Hz}) .{ }^{1} \mathrm{H}$ NMR data are reported as follows: chemical shift $(\mathrm{ppm})$, multiplicity $(\mathrm{s}=$ singlet, $\mathrm{d}=$ doublet, $\mathrm{dd}=$ doublet of doublets, $\mathrm{dt}=$ doublet of triplets, $\mathrm{dtdq}=$ doublet of triplet of doublet of quartets, $\mathrm{dq}=$ doublet of quartets, $\mathrm{ddd}=$ doublet of doublet of doublets, $\mathrm{ddt}=$ doublet of doublets of triplets, dddd $=$ doublet of doublets of doublets of doublets, ddquint $=$ doublet of doublet of quintets, dddquint $=$ doublet of doublet of doublet of quintets, quint $=$ quintet, $\mathrm{t}=$ triplet, $\mathrm{td}=$ triplet of doublets, $\mathrm{tt}=$ triplet of triplets $\mathrm{m}=$ multiplet, $\mathrm{b}=$ broad), coupling constants $(\mathrm{Hz})$, integration and assignment. Compounds were fully characterized by ${ }^{1} \mathrm{H},{ }^{13} \mathrm{C}, 2 \mathrm{D}$ gDQCOSY, gHSQC, gHMBC, NOESY, and HOESY spectra. The characterization and assignment of isoxazolidines $4 \mathbf{a}-\mathbf{r}$ were done either from isolated compounds or mixtures of isomers. Column or flash chromatography was carried out using ACROS silica gel $(0.035-0.070 \mathrm{~mm}$, and $60 \AA$ pore diameter $)$.

General Procedure for the Synthesis of Trifluoromethyl Vinyl Sulfide 2. 2-Chloroethyl trifluoromethyl sulfide (1.0 equiv) was dissolved in distilled THF $(0.40 \mathrm{M})$ under inert atmosphere and cooled to $0{ }^{\circ} \mathrm{C}$. Then, $\mathrm{KO}^{t} \mathrm{Bu}$ (1.0 equiv, $1.0 \mathrm{M}$ solution in THF) was added slowly, and the mixture was warmed to $21^{\circ} \mathrm{C}$ for $90 \mathrm{~min}$. This solution was used for the cycloaddition reactions of alkene $2 .{ }^{1} \mathrm{H}$ NMR [400 MHz, $\delta$ (ppm), THF- $d_{8}$ ]: $6.54(\mathrm{dd}, J=16.5,9.4 \mathrm{~Hz}, 1 \mathrm{H}), 5.75-5.66$ $(\mathrm{m}, 2 \mathrm{H}) .{ }^{13} \mathrm{C}$ NMR [101 MHz, $\delta$ (ppm), THF- $d_{8}$ ]: $129.8(\mathrm{q}, J=306.5$ $\mathrm{Hz}), 124.4(\mathrm{q}, J=1.0 \mathrm{~Hz}), 121.3(\mathrm{q}, J=3.2 \mathrm{~Hz}) .{ }^{19} \mathrm{~F}$ NMR [377 MHz, $\delta$ (ppm), THF- $\left.d_{8}\right]:-43.6$.

General Procedure for the Synthesis of Hydroxylamines $5 \mathrm{~b}$ and 5c. The corresponding nitrobenzene (12b or 12c; 1 equiv) was added to a flame-dried Schlenk tube containing a suspension of Pd/C ( 0.25 equiv) in dry THF $(10 \mathrm{~mL})$. The mixture was cooled to $0{ }^{\circ} \mathrm{C}$, and $\mathrm{N}_{2} \mathrm{H}_{4} \cdot \mathrm{H}_{2} \mathrm{O}$ (2.0 equiv) was added dropwise. The reaction was carefully monitored by TLC until the starting material disappeared. The crude mixture was diluted with THF $(10 \mathrm{~mL})$ and filtered over a pad of diatomaceous earth. The solvent was evaporated in vacuo, and the crude mixture was purified by column chromatography using $\mathrm{CH}_{2} \mathrm{Cl}_{2}$ under a nitrogen atmosphere.

$\mathrm{N}$-[3-(Trifluoromethyl)phenyl]hydroxylamine (5b). According to general procedure, the reaction of 1-nitro-3-(trifluoromethyl)benzene 12b $(1.0 \mathrm{~g}, 5.23 \mathrm{mmol})$ afforded hydroxylamine $5 \mathbf{b}(885 \mathrm{mg}, 5.00$ mmol) as a pale yellow oil. ${ }^{1} \mathrm{H}$ NMR $\left[400 \mathrm{MHz}, \delta(\mathrm{ppm}), \mathrm{CDCl}_{3}\right]$ : 7.41-7.34 (m, $1 \mathrm{H}), 7.28-7.26(\mathrm{~m}, 1 \mathrm{H}), 7.23-7.19(\mathrm{~m}, 1 \mathrm{H}), 7.15-$ $7.10(\mathrm{~m}, 1 \mathrm{H}), 6.74-4.98$ (bs, $2 \mathrm{H}) . R_{f}: 0.31\left(\mathrm{CH}_{2} \mathrm{Cl}_{2}\right)$. Yield: $95 \%$. NMR spectral data are in accordance with previously reported data. ${ }^{20}$

$\mathrm{N}$-(3,5-Dichlorophenyl)hydroxylamine (5c). According to general procedure, the reaction of 1,3-dichloro-5-nitrobenzene $12 \mathrm{c}(1.0 \mathrm{~g}, 5.22$ mmol) afforded hydroxylamine $5 \mathrm{c}(827 \mathrm{mg}, 4.65 \mathrm{mmol})$ as a yellow solid. ${ }^{1} \mathrm{H}$ NMR $\left[400 \mathrm{MHz}, \delta(\mathrm{ppm}), \mathrm{CDCl}_{3}\right]: 6.93(\mathrm{t}, J=1.8 \mathrm{~Hz}, 1 \mathrm{H})$, $6.88(\mathrm{~d}, J=1.8 \mathrm{~Hz}, 2 \mathrm{H}), 6.79(\mathrm{bs}, 1 \mathrm{H}), 5.31$ (bs, $1 \mathrm{H}) . R_{f}: 0.33$ $\left(\mathrm{CH}_{2} \mathrm{Cl}_{2}\right)$. Yield: $89 \%$. NMR spectral data are in accordance with previously reported data. ${ }^{20}$

General Procedure for the Synthesis of Isoxazolidines 3a-c. A solution of the corresponding hydroxylamine 5 (1 equiv) in THF $(0.22 \mathrm{M})$ was added to a solution of paraformaldehyde (3 equiv) in THF $(0.22 \mathrm{M})$ at $0{ }^{\circ} \mathrm{C}$. Then a cooled solution $\left(0{ }^{\circ} \mathrm{C}\right)$ of alkene $2(6.0$ equiv) was subsequently added to the solution containing paraformaldehyde and the hydroxylamine. Finally, a solution of $\operatorname{In}(\mathrm{OTf})_{3}(0.06$ equiv) in dry THF ( $44 \mathrm{mM})$ was added to the reaction mixture. The reaction mixture was warmed to $21^{\circ} \mathrm{C}$ and stirred for $16 \mathrm{~h}$. Then, the reaction mixture was quenched with a saturated aqueous solution of $\mathrm{NaHCO}_{3}(10 \mathrm{~mL})$, and the aqueous layer was extracted with AcOEt (2 $\times 10 \mathrm{~mL})$. The combined organic layers were dried over $\mathrm{MgSO}_{4}$ filtered off and concentrated in vacuo. The crude mixture was purified by column chromatography using the indicated eluent to afford isoxazolines $3 \mathbf{a}-\mathbf{c}$.

2-Phenyl-5-[(trifluoromethyl)sufanyl]isoxazolidine (3a). According to the general procedure, the reaction of $N$-phenylhydroxylamine $5 a(17.8 \mathrm{mg}, 0.163 \mathrm{mmol})$ afforded isoxazolidine 3a $(15.3 \mathrm{mg}, 0.163$ $\mathrm{mmol}$ ) as a brown solid after column chromatography (heptane/ AcOEt, 19:1 $\rightarrow$ 4:1). ${ }^{1} \mathrm{H}$ NMR [500 MHz, $\delta(\mathrm{ppm}), \mathrm{CDCl}_{3}$ ]: 7.34- 
$7.27(\mathrm{~m}, 2 \mathrm{H}), 7.08-7.05(\mathrm{~m}, 2 \mathrm{H}), 7.03(\mathrm{tt}, J=7.3,1.1 \mathrm{~Hz}, 1 \mathrm{H}), 5.98$ (dd, $J=8.1,4.3 \mathrm{~Hz}, 1 \mathrm{H}), 3.85-3.71(\mathrm{~m}, 1 \mathrm{H}), 3.24$ (ddd, $J=9.3,8.2$, $7.6 \mathrm{~Hz}, 1 \mathrm{H}), 2.91(\mathrm{dtdq}, J=13.2,8.2,4.0,0.8 \mathrm{~Hz}, 1 \mathrm{H}), 2.48-2.28(\mathrm{~m}$, $1 \mathrm{H}) .{ }^{13} \mathrm{C}$ NMR $\left[126 \mathrm{MHz}, \delta(\mathrm{ppm}), \mathrm{CDCl}_{3}\right]: 149.8,130.1(\mathrm{q}, J=$ $307.7 \mathrm{~Hz}), 129.0,123.0,116.1,81.1(\mathrm{q}, J=2.2 \mathrm{~Hz}), 52.0,36.0(\mathrm{q}, J=1.1$ $\mathrm{Hz}) .{ }^{19} \mathrm{~F}$ NMR $\left[471 \mathrm{MHz}, \delta(\mathrm{ppm}), \mathrm{CDCl}_{3}\right]:-39.8$. FTIR $\left[\bar{\nu}\left(\mathrm{cm}^{-1}\right)\right]$ : 2860, 1599, 1491, 1295, 1109, 752, 692. HRMS (EI) $\mathrm{m} / z:\left[\mathrm{M}^{+\bullet}\right]$ calcd for $\mathrm{C}_{10} \mathrm{H}_{10} \mathrm{~F}_{3} \mathrm{NOS} 249.0435$, found 249.0458. $R_{f}: 0.56$ (heptane/ AcOEt, 4:1). Yield: $38 \%$.

2-[3-(Trifluoromethyl)phenyl]-5-[(trifluoromethyl)sulfanyl]isoxazolidine ( $3 b)$. According to the general procedure, the reaction of hydroxylamine $\mathbf{5 b}(56.2 \mathrm{mg}, 0.318 \mathrm{mmol})$ afforded isoxazolidine $\mathbf{3 b}$ (23.5 $\mathrm{mg}, 0.071 \mathrm{mmol}$ ) as a colorless oil, after column chromatography (heptane/AcOEt $\left./ \mathrm{Et}_{3} \mathrm{~N}, 12.2: 1: 0.13\right) .{ }^{1} \mathrm{H}$ NMR [500 MHz, $\delta(\mathrm{ppm})$, $\mathrm{CDCl}_{3}$ ]: 7.41 (ddquint, $\left.J=8.2,7.2,0.8 \mathrm{~Hz}, 1 \mathrm{H}\right), 7.29-7.25(\mathrm{~m}, 2 \mathrm{H})$, $7.23-7.19(\mathrm{~m}, 1 \mathrm{H}), 6.01(\mathrm{dd}, J=8.0,4.2 \mathrm{~Hz}, 1 \mathrm{H}), 3.83(\mathrm{ddd}, J=9.1$, 8.2, $4.3 \mathrm{~Hz}, 1 \mathrm{H}), 3.30(\mathrm{ddd}, J=9.1,8.5,7.4 \mathrm{~Hz}, 1 \mathrm{H}), 3.02-2.90(\mathrm{~m}, 1$ $\mathrm{H}), 2.48-2.38(\mathrm{~m}, 1 \mathrm{H}) .{ }^{13} \mathrm{C}$ NMR $\left[126 \mathrm{MHz}, \delta(\mathrm{ppm}), \mathrm{CDCl}_{3}\right]$ : $150.1,131.3(\mathrm{q}, J=32.3 \mathrm{~Hz}), 129.8(\mathrm{q}, J=307.9 \mathrm{~Hz}), 129.4,124.0(\mathrm{q}, J$ $=272.4 \mathrm{~Hz}), 119.2(\mathrm{q}, J=3.8 \mathrm{~Hz}), 118.9,112.3(\mathrm{q}, J=4.0 \mathrm{~Hz}), 81.1(\mathrm{q}$, $J=2.4 \mathrm{~Hz}), 51.7,35.8(\mathrm{q}, J=1.2 \mathrm{~Hz}) .{ }^{19} \mathrm{~F}$ NMR $[471 \mathrm{MHz}, \delta(\mathrm{ppm})$, $\left.\mathrm{CDCl}_{3}\right]:-39.9,-62.8$. FTIR $\left[\bar{\nu}\left(\mathrm{cm}^{-1}\right)\right]: 2956,2925,2855,1593$, $1328,1120,795,698$. HRMS (EI) $m / z:\left[\mathrm{M}^{+\bullet}\right]$ calcd for $\mathrm{C}_{11} \mathrm{H}_{9} \mathrm{~F}_{6} \mathrm{NOS}$ 317.0309, found 317.0325. $R_{f}: 0.44$ (heptane/AcOEt, 4:1). Yield: $22 \%$.

2-(3,5-Dichlorophenyl)-5-[(trifluoromethyl)sulfanyl]isoxazolidine (3c). According to the general procedure, the reaction of hydroxylamine $5 c(29.3 \mathrm{mg}, 0.166 \mathrm{mmol})$ afforded isoxazolidine $3 \mathrm{c}(10.33 \mathrm{mg}, 0.033$ $\mathrm{mmol})$ as a green oil after column chromatography (heptane/AcOEt/ $\left.\mathrm{Et}_{3} \mathrm{~N}, 12.2: 1: 0.13\right) .{ }^{1} \mathrm{H}$ NMR [500 MHz, $\left.\delta(\mathrm{ppm}), \mathrm{CDCl}_{3}\right]: 6.99(\mathrm{t}, J=$ $1.8 \mathrm{~Hz}, 1 \mathrm{H}), 6.91(\mathrm{~d}, J=1.8 \mathrm{~Hz}, 2 \mathrm{H}), 5.98(\mathrm{dd}, J=8.0,4.1 \mathrm{~Hz}, 1 \mathrm{H})$, 3.75 (ddd, $J=8.9,8.1,4.4 \mathrm{~Hz}, 1 \mathrm{H}$ ), 3.27 (ddd, $J=9.2,8.6,7.1 \mathrm{~Hz}, 1$ $\mathrm{H}), 3.01-2.87(\mathrm{~m}, 1 \mathrm{H}), 2.47-2.36(\mathrm{~m}, 1 \mathrm{H}) .{ }^{13} \mathrm{C}$ NMR [126 MHz, $\delta$ (ppm), $\left.\mathrm{CDCl}_{3}\right]: 151.6,135.4,129.7(\mathrm{q}, J=307.9 \mathrm{~Hz}), 122.5,114.2$, $81.2(\mathrm{q}, J=2.6 \mathrm{~Hz}), 51.6,35.8(\mathrm{q}, J=1.1 \mathrm{~Hz})$. FTIR $\left[\bar{\nu}\left(\mathrm{cm}^{-1}\right)\right]: 2924$, 2850, 1587, 1260, 1115, 1051, 796. HRMS (EI) $\mathrm{m} / z:\left[\mathrm{M}^{+\bullet}\right]$ calcd for $\mathrm{C}_{10} \mathrm{H}_{8} \mathrm{Cl}_{2} \mathrm{~F}_{3} \mathrm{NOS}$ 316.9656, found 316.9657. $R_{f} 0.45$ (heptane/AcOEt, 4:1). Yield: $20 \%$.

General Procedure for the Synthesis of Isoxazolidines 3d and 3e. A solution of hydroxylamine hydrochloride $5 \mathrm{~d}$ or $5 \mathrm{e}$ ( 1 equiv) in THF $(0.22 \mathrm{M})$ and $\mathrm{Et}_{3} \mathrm{~N}$ (1 equiv) was added to a solution of paraformaldehyde ( 3 equiv) in THF $(0.22 \mathrm{M})$ at $0{ }^{\circ} \mathrm{C}$. Then a cooled solution $\left(0^{\circ} \mathrm{C}\right)$ of alkene 2 (6.0 equiv) was subsequently added to the solution containing paraformaldehyde and the hydroxylamine. Finally, a solution of $\operatorname{In}(\mathrm{OTf})_{3}(0.06$ equiv) in dry THF $(22 \mathrm{mM})$ was added to the reaction mixture. The reaction mixture was warmed to $21{ }^{\circ} \mathrm{C}$ and stirred for $16 \mathrm{~h}$. Then, the reaction mixture was quenched with a saturated aqueous solution of $\mathrm{NaHCO}_{3}(10 \mathrm{~mL})$, and the aqueous layer was extracted with AcOEt $(2 \times 10 \mathrm{~mL})$. The combined organic layers were dried over $\mathrm{MgSO}_{4}$, filtered off, and concentrated in vacuo. The crude mixture was purified by column chromatography using the indicated eluent to afford isoxazolines $\mathbf{3 d}$ and $\mathbf{3 e}$.

2-Benzyl-5-[(trifluoromethyl)sulfanyl]isoxazolidine (3d). According to the general procedure, the reaction of hydroxylamine hydrochloride $\mathbf{5 d}(26.8 \mathrm{mg}, 0.169 \mathrm{mmol})$ afforded isoxazolidine $\mathbf{3 d}$ (13.2 $\mathrm{mg}, 0.050 \mathrm{mmol}$ ) as a colorless oil after column chromatography (heptane/AcOEt $\left./ \mathrm{Et}_{3} \mathrm{~N}, 9: 1: 0.1\right) .{ }^{1} \mathrm{H}$ NMR $[500 \mathrm{MHz}, \delta(\mathrm{ppm})$, $\mathrm{CDCl}_{3}$ ]: 7.40-7.36 (m, $\left.2 \mathrm{H}\right), 7.35-7.30(\mathrm{~m}, 2 \mathrm{H}), 7.30-7.27(\mathrm{~m}, 1$ $\mathrm{H}), 5.86(\mathrm{dd}, J=8.3,4.4 \mathrm{~Hz}, 1 \mathrm{H}), 4.35-3.82(\mathrm{~m}, 2 \mathrm{H}), 3.34-3.11(\mathrm{~m}$, $1 \mathrm{H}), 3.11-2.40(\mathrm{~m}, 1 \mathrm{H}), 2.83(\mathrm{dtd}, J=11.9,8.0,3.3 \mathrm{~Hz}, 1 \mathrm{H}), 2.40-$ $2.18(\mathrm{~m}, 1 \mathrm{H}) .{ }^{13} \mathrm{C}$ NMR $\left[126 \mathrm{MHz}, \delta(\mathrm{ppm}), \mathrm{CDCl}_{3}\right]: 136.2,130.3(\mathrm{q}$, $J=307.9 \mathrm{~Hz}), 128.9,128.4,127.5,81.3(\mathrm{q}, J=2.4 \mathrm{~Hz}), 61.7,53.3,36.5$. ${ }^{19} \mathrm{~F}$ NMR [471 MHz, $\left.\delta(\mathrm{ppm}), \mathrm{CDCl}_{3}\right]:-39.9$. FTIR $\left[\bar{\nu}\left(\mathrm{cm}^{-1}\right)\right]$ : $2849,1606,1497,1455,1117,755,698$. HRMS (EI) $m / z:\left[\mathrm{M}^{+\bullet}\right]$ calcd for $\mathrm{C}_{11} \mathrm{H}_{12} \mathrm{~F}_{3} \mathrm{NOS}$ 263.0592, found 263.0604. $R_{f}: 0.41$ (heptane/ AcOEt, 4:1). Yield: $30 \%$.

2-Cyclohexyl-5-[(trifluoromethyl)sulfanyl]isoxazolidine (3e). According to the general procedure, the reaction of hydroxylamine hydrochloride $5 \mathrm{e}(23.1 \mathrm{mg}, 0.152 \mathrm{mmol})$ afforded isoxazolidine $3 \mathrm{e}$ (20.4 $\mathrm{mg}, 0.080 \mathrm{mmol}$ ) as a colorless oil, after column chromatography (heptane/AcOEt, 19:1 $\rightarrow$ 5:1). ${ }^{1} \mathrm{H}$ NMR [400 MHz, $\left.\delta(\mathrm{ppm}), \mathrm{CDCl}_{3}\right]$ : $5.82(\mathrm{dd}, J=8.1,4.2 \mathrm{~Hz}, 1 \mathrm{H}), 3.43-3.13(\mathrm{~m}, 1 \mathrm{H}), 2.85-2.73(\mathrm{~m}, 1$ H), 2.68-2.50 (m, $1 \mathrm{H}), 2.68-2.18(\mathrm{~m}, 1 \mathrm{H}), 2.31-2.18(\mathrm{~m}, 1 \mathrm{H})$, $1.83-1.69(\mathrm{~m}, 2 \mathrm{H}), 1.66-1.52(\mathrm{~m}, 2 \mathrm{H}), 1.39-1.13(\mathrm{~m}, 6 \mathrm{H}) .{ }^{13} \mathrm{C}$ NMR [101 MHz, $\left.\delta(\mathrm{ppm}), \mathrm{CDCl}_{3}\right]: 130.4(\mathrm{q}, J=308.1 \mathrm{~Hz}), 80.8(\mathrm{q}, J$ $=2.2 \mathrm{~Hz}), 65.8,51.5,36.6,26.0,24.8,24.4 .{ }^{19} \mathrm{~F}$ NMR $[377 \mathrm{MHz}, \delta$ $\left.(\mathrm{ppm}), \mathrm{CDCl}_{3}\right]:-40.0$. FTIR $\left[\bar{\nu}\left(\mathrm{cm}^{-1}\right)\right]: 2924,2854,1728,1455$, 1258, 1082, 1009, 788. $R_{f} 0.44$ (heptane/AcOEt, 9:1). Yield: 53\%.

General Procedure A for the Syntheses of Nitrones $6 \mathrm{~d}-\mathrm{g}, \mathbf{6 i}$, $6 \mathbf{6 j}, 6 \mathbf{I}-\mathbf{r}$. The corresponding aldehyde (1.0 equiv, dissolved in $1.5 \mathrm{~mL}$ of dry EtOH for solid compounds) was added to a solution of $\mathrm{N}$ phenylhydroxylamine (1.05 equiv) in dry $\mathrm{EtOH}(1.0 \mathrm{~mL})$ at $21{ }^{\circ} \mathrm{C}$ under a nitrogen atmosphere. The reaction mixture was stirred for 16$21 \mathrm{~h}$, and then the solvent was removed under vacuo. The crude mixture was purified by column chromatography using the indicated eluent to afford nitrones $\mathbf{6} \mathbf{d}-\mathrm{g}, \mathbf{6 i}, \mathbf{6} \mathbf{j}, \mathbf{6} \mathbf{\mathbf { l }}-\mathbf{r}$.

General Procedure B for the Syntheses of Nitrones $6 \mathrm{a}-\mathrm{c}, \mathbf{6 h}$, and $6 \mathrm{k}$. The corresponding aldehyde (1.0 equiv) was added to a solution of $\mathrm{N}$-phenylhydroxylamine (1.0 equiv) in dry EtOH $(1.0 \mathrm{~mL})$, and the flask was wrapped with aluminum foil. The reaction mixture was stirred at $21^{\circ} \mathrm{C}$ for $16-21 \mathrm{~h}$, and then the solvent was removed under vacuo. The crude mixture was purified by column chromatography using the indicated eluent to afford nitrones $\mathbf{6 a}-\mathbf{c}, \mathbf{6 h}$, and $\mathbf{6 k}$.

(Z)-N,1-Diphenylmethanimine Oxide (6a). ${ }^{21}$ According to general procedure $\mathrm{B}$, the reaction of benzaldehyde $(47.0 \mu \mathrm{L}, 0.458 \mathrm{mmol})$ with $\mathrm{N}$-phenylhydroxylamine $(50 \mathrm{mg}, 0.458 \mathrm{mmol})$ afforded nitrone $\mathbf{6 a}$ (71 $\mathrm{mg}, 0.360 \mathrm{mmol}$ ) as a white solid, after column chromatography (heptane/AcOEt, 5:1 $\rightarrow$ 1:1). ${ }^{1} \mathrm{H}$ NMR $\left[400 \mathrm{MHz}, \delta(\mathrm{ppm}), \mathrm{CDCl}_{3}\right]$ : 8.44-8.36 (m, $2 \mathrm{H}), 7.92(\mathrm{~s}, 1 \mathrm{H}), 7.81-7.74(\mathrm{~m}, 2 \mathrm{H}), 7.53-7.43(\mathrm{~m}$, $6 \mathrm{H})$. Yield: $79 \%$

(Z)-1-(4-Fluorophenyl)-N-phenylmethanimine Oxide (6b). ${ }^{22}$ According to general procedure $\mathrm{B}$, the reaction of 4-fluorobenzaldehyde $(98.0 \mu \mathrm{L}, 0.916 \mathrm{mmol})$ with $N$-phenylhydroxylamine $(100 \mathrm{mg}, 0.916$ $\mathrm{mmol}$ ) afforded nitrone $\mathbf{6 b}(178.5 \mathrm{mg}, 0.829 \mathrm{mmol})$ as a white solid. ${ }^{1} \mathrm{H}$ $\mathrm{NMR}\left[400 \mathrm{MHz}, \delta(\mathrm{ppm}), \mathrm{CDCl}_{3}\right]: 8.49-8.42(\mathrm{~m}, 2 \mathrm{H}), 7.91(\mathrm{~s}, 1 \mathrm{H})$, 7.80-7.75 (m, $2 \mathrm{H}), 7.55-7.44(\mathrm{~m}, 3 \mathrm{H}), 7.23-7.14$ (m, $2 \mathrm{H})$. Yield: $91 \%$.

(Z)-N-Phenyl-1-(4-tolyl)methanimine Oxide (6c). ${ }^{22}$ According to general procedure $\mathrm{B}$, the reaction of 4-methylbenzaldehyde $(163.0 \mu \mathrm{L}$, $1.375 \mathrm{mmol})$ with $\mathrm{N}$-phenylhydroxylamine $(127 \mathrm{mg}, 1.375 \mathrm{mmol})$ afforded nitrone $6 \mathrm{c}(267.2 \mathrm{mg}, 1.26 \mathrm{mmol})$ as yellow crystals, after column chromatography (heptane/AcOEt, 5:1 $\rightarrow 1: 1$ ). ${ }^{1} \mathrm{H}$ NMR [400 $\mathrm{MHz}, \delta(\mathrm{ppm}), \mathrm{CDCl}_{3}$ ]: 8.34-8.27 (m, $\left.2 \mathrm{H}\right), 7.89(\mathrm{~s}, 1 \mathrm{H}), 7.81-7.75$ $(\mathrm{m}, 2 \mathrm{H}), 7.51-7.43(\mathrm{~m}, 3 \mathrm{H}), 7.33-7.28(\mathrm{~m}, 2 \mathrm{H}), 2.42(\mathrm{~s}, 3 \mathrm{H})$. Yield: $92 \%$.

(Z)-N-Phenyl-1-[4-(trifluoromethyl)phenyl]methanimine oxide (6d). ${ }^{23}$ According to general procedure A, the reaction of 4(trifluoromethyl)benzaldehyde $(91 \mathrm{mg}, 0.523 \mathrm{mmol})$ with $\mathrm{N}$-phenylhydroxylamine $(60 \mathrm{mg}, 0.549 \mathrm{mmol})$ afforded nitrone $6 \mathrm{~d}(139 \mathrm{mg}$, $0.523 \mathrm{mmol})$ as a yellow solid. ${ }^{1} \mathrm{H} \mathrm{NMR}\left[400 \mathrm{MHz}, \delta(\mathrm{ppm}), \mathrm{CDCl}_{3}\right]$ : 8.54-8.48 (m, $2 \mathrm{H}), 8.00(\mathrm{~s}, 1 \mathrm{H}), 7.83-7.76(\mathrm{~m}, 2 \mathrm{H}), 7.76-7.70(\mathrm{~m}$, $2 \mathrm{H}), 7.55-7.47(\mathrm{~m}, 3 \mathrm{H})$. Yield: $99 \%$.

(Z)-1-[4-(Pentafluoro- $\lambda^{6}$-sulfanyl)phenyl]-N-phenylmethanimine Oxide (6e). According to general procedure A, the reaction of 4(pentafluoro- $\lambda^{6}$-sulfanyl)benzaldehyde $(120 \mathrm{mg}, 0.517 \mathrm{mmol})$ with $N$ phenylhydroxylamine $(59 \mathrm{mg}, 0.543 \mathrm{mmol})$ afforded nitrone $6 \mathrm{e}(163.3$ $\mathrm{mg}, 0.505 \mathrm{mmol})$ as a white-yellow solid. ${ }^{1} \mathrm{H}$ NMR $[400 \mathrm{MHz}, \delta$ (ppm), $\left.\mathrm{CDCl}_{3}\right]: 8.52-8.45(\mathrm{~m}, 2 \mathrm{H}), 8.00(\mathrm{~s}, 1 \mathrm{H}), 7.89-7.81(\mathrm{~m}, 2$ $\mathrm{H}), 7.81-7.74(\mathrm{~m}, 2 \mathrm{H}), 7.58-7.46(\mathrm{~m}, 3 \mathrm{H}) .{ }^{13} \mathrm{C}$ NMR $[101 \mathrm{MHz}, \delta$ (ppm), $\mathrm{CDCl}_{3}$ ]: 154.5, 149.1, 133.6, 132.5, 130.7, 129.5, 128.9, 126.5 (quint, $J=4.4 \mathrm{~Hz}$ ), 121.9. FTIR $\left[\bar{\nu}\left(\mathrm{cm}^{-1}\right)\right]: 3057,1594,1573,1074$, 821, 810, 765. HRMS (ESI-TOF) $\mathrm{m} / z$ : $[\mathrm{M}+\mathrm{H}]^{+}$calcd for $\mathrm{C}_{13} \mathrm{H}_{11} \mathrm{~F}_{5} \mathrm{NOS}$ 324.0476, found 324.0482. $R_{f}$. $0.45\left(\mathrm{CH}_{2} \mathrm{Cl}_{2}\right)$. Yield: 98\%.

(Z)-1-(4-Methoxyphenyl)-N-phenylmethanimine oxide (6f). ${ }^{24} \mathrm{Ac}$ cording to general procedure $\mathrm{A}$, the reaction of 4-methoxybenzaldehyde $(0.063 \mathrm{~mL}, 0.514 \mathrm{mmol})$ with $\mathrm{N}$-phenylhydroxylamine $(59 \mathrm{mg}$, $0.540 \mathrm{mmol})$ afforded nitrone $6 \mathrm{f}(110 \mathrm{mg}, 0.484 \mathrm{mmol})$ as a yellowbrown solid, after column chromatography $\left(\mathrm{CH}_{2} \mathrm{Cl}_{2} \rightarrow \mathrm{CH}_{2} \mathrm{Cl}_{2}\right.$ / $\mathrm{MeOH}, 10: 0.2) .{ }^{1} \mathrm{H}$ NMR $\left[400 \mathrm{MHz}, \delta\right.$ (ppm), $\mathrm{CDCl}_{3}$ ]: 8.44-8.36 
(m, $2 \mathrm{H}), 7.84(\mathrm{~s}, 1 \mathrm{H}), 7.79-7.72(\mathrm{~m}, 2 \mathrm{H}), 7.50-7.37(\mathrm{~m}, 3 \mathrm{H})$, 7.01-6.93 (m, $2 \mathrm{H}), 3.86(\mathrm{~s}, 3 \mathrm{H})$. Yield: $94 \%$.

(Z)-1-(3-Fluorophenyl)-N-phenylmethanimine Oxide (6g). ${ }^{25} \mathrm{Ac}$ cording to general procedure $A$, the reaction of 3-fluorobenzaldehyde $(0.060 \mathrm{~mL}, 0.564 \mathrm{mmol})$ with $\mathrm{N}$-phenylhydroxylamine $(62 \mathrm{mg}, 0.564$ mmol) afforded nitrone $6 \mathrm{~g}(118 \mathrm{mg}, 0.548 \mathrm{mmol})$ as a light brown solid, after column chromatography (heptane/ $\mathrm{CH}_{2} \mathrm{Cl}_{2}, 1: 4 \rightarrow \mathrm{CH}_{2} \mathrm{Cl}_{2} /$ $\mathrm{MeOH}, 10: 1) .{ }^{1} \mathrm{H}$ NMR [400 MHz, $\delta$ (ppm), $\mathrm{CDCl}_{3}$ ]: 8.44 (ddd, $J=$ 10.6, 2.6, $1.6 \mathrm{~Hz}, 1 \mathrm{H}), 7.94(\mathrm{~s}, 1 \mathrm{H}), 7.90$ (ddt, $J=8.1,1.6,0.7 \mathrm{~Hz}, 1$ H), 7.80-7.73 (m, $2 \mathrm{H}), 7.54-7.47(\mathrm{~m}, 3 \mathrm{H}), 7.44(\mathrm{td}, J=8.1,5.9 \mathrm{~Hz}$, $1 \mathrm{H}), 7.17(\mathrm{tdd}, J=8.1,2.6,1.0 \mathrm{~Hz}, 1 \mathrm{H}) .{ }^{13} \mathrm{C} \mathrm{NMR}[101 \mathrm{MHz}, \delta$ (ppm), $\left.\mathrm{CDCl}_{3}\right]: 162.8(\mathrm{~d}, J=245.5 \mathrm{~Hz}), 149.1,133.5,132.6(\mathrm{~d}, J=9.1$ $\mathrm{Hz}), 130.4,130.1(\mathrm{~d}, J=8.4 \mathrm{~Hz}), 129.4,125.1(\mathrm{~d}, J=3.0 \mathrm{~Hz}), 121.8$, $118.0(\mathrm{~d}, J=21.7 \mathrm{~Hz}), 115.3(\mathrm{~d}, J=24.7 \mathrm{~Hz})$. Yield: $97 \%$.

(Z)-N-Phenyl-1-(3-tolyl)methanimine Oxide $(6 h) .{ }^{25}$ According to general procedure $\mathrm{B}$, the reaction of 3-methylbenzaldehyde ( $42 \mu \mathrm{L}$, $0.458 \mathrm{mmol}$ ) with $\mathrm{N}$-phenylhydroxylamine $(50 \mathrm{mg}, 0.458 \mathrm{mmol})$ afforded nitrone $\mathbf{6 h}(95.3 \mathrm{mg}, 0.451 \mathrm{mmol})$ as a yellow oil, after column chromatography (heptane/AcOEt, 5:1 $\rightarrow 1: 1) .{ }^{1} \mathrm{H}$ NMR $[400 \mathrm{MHz}, \delta$ (ppm), $\mathrm{CDCl}_{3}$ ]: 8.32 (tq $\left.J=1.8,0.7 \mathrm{~Hz}, 1 \mathrm{H}\right), 8.12$ (dddquint, $J=7.8$, $1.8,1.2,0.7 \mathrm{~Hz}, 1 \mathrm{H}), 7.89(\mathrm{~s}, 1 \mathrm{H}), 7.81-7.75(\mathrm{~m}, 2 \mathrm{H}), 7.53-7.44(\mathrm{~m}$, $3 \mathrm{H}), 7.38(\mathrm{t}, J=7.8 \mathrm{~Hz}, 1 \mathrm{H}), 7.32-7.27(\mathrm{~m}, 1 \mathrm{H}), 2.43(\mathrm{~s}, 3 \mathrm{H}) .{ }^{13} \mathrm{C}$ NMR [101 MHz, $\delta$ (ppm), $\mathrm{CDCl}_{3}$ ]: 149.3, 138.5, 134.9, 132.0, 130.8, 130.0, 129.4, 129.3, 128.7, 126.6, 121.9, 21.6. FTIR $\left[\bar{\nu}\left(\mathrm{cm}^{-1}\right)\right]: 3092$, 2963, 2920, 1652, 1590, 1459, 1088, 781, 692. HRMS (ESI-TOF) $\mathrm{m} / z$ : $[\mathrm{M}+\mathrm{H}]^{+}$calcd for $\mathrm{C}_{14} \mathrm{H}_{14} \mathrm{NO} 212.1070$, found 212.1085 . Yield: $98 \%$.

(Z)-1-[3-(Methoxycarbonyl)phenyl]-N-phenylmethanimine Oxide (6i). According to general procedure A, the reaction of methyl 3formylbenzoate $(90 \mathrm{mg}, 0.548 \mathrm{mmol}$ ) with $N$-phenylhydroxylamine $(63 \mathrm{mg}, 0.576 \mathrm{mmol})$ afforded nitrone $6 \mathbf{i}(112 \mathrm{mg}, 0.439 \mathrm{mmol})$ as a yellow oil, after column chromatography $\left(\mathrm{CH}_{2} \mathrm{Cl}_{2} \rightarrow \mathrm{CH}_{2} \mathrm{Cl}_{2} / \mathrm{MeOH}\right.$, 10:1). ${ }^{1} \mathrm{H}$ NMR $\left[400 \mathrm{MHz}, \delta(\mathrm{ppm}), \mathrm{CDCl}_{3}\right]: 8.88$ (dddd, $J=7.9,1.7$, $1.2,0.5 \mathrm{~Hz}, 1 \mathrm{H}), 8.80(\mathrm{tt}, J=1.7,0.5 \mathrm{~Hz}, 1 \mathrm{H}), 8.13$ (ddd, $J=7.9,1.7$, $1.2 \mathrm{~Hz}, 1 \mathrm{H}), 8.01(\mathrm{~d}, J=0.5 \mathrm{~Hz}, 1 \mathrm{H}), 7.83-7.75(\mathrm{~m}, 2 \mathrm{H}), 7.59(\mathrm{tt}, J=$ 7.9, $0.5 \mathrm{~Hz}, 1 \mathrm{H}), 7.54-7.46(\mathrm{~m}, 3 \mathrm{H}), 3.95(\mathrm{~s}, 3 \mathrm{H}) .{ }^{13} \mathrm{C}$ NMR $[101$ $\left.\mathrm{MHz}, \delta(\mathrm{ppm}), \mathrm{CDCl}_{3}\right]: 166.5,149.0,133.6,132.6,131.6,131.0,130.6$, 130.4, 130.2, 129.3, 129.0, 121.7, 52.4. FTIR $\left[\bar{\nu}\left(\mathrm{cm}^{-1}\right)\right]: 3064,2952$, $1718,1434,1278,1187,1072,912,729,684$. HRMS (ESI-TOF) $\mathrm{m} / z$ : $[\mathrm{M}+\mathrm{H}]^{+}$calcd for $\mathrm{C}_{15} \mathrm{H}_{14} \mathrm{NO}_{3}$ 256.0968, found 256.0984. $R_{f}: 0.07$ $\left(\mathrm{CH}_{2} \mathrm{Cl}_{2}\right)$. Yield: $80 \%$.

(Z)-1-(3-Methoxyphenyl)-N-phenylmethanimine Oxide (6j). According to general procedure $\mathrm{A}$, the reaction of 3-methoxybenzaldehyde $(0.063 \mathrm{~mL}, 0.514 \mathrm{mmol})$ with $\mathrm{N}$-phenylhydroxylamine $(59 \mathrm{mg}$, $0.540 \mathrm{mmol}$ ) afforded nitrone $6 \mathbf{j}(115 \mathrm{mg}, 0.506 \mathrm{mmol})$ as a yellow oil, after column chromatography $\left(\mathrm{CH}_{2} \mathrm{Cl}_{2} \rightarrow \mathrm{CH}_{2} \mathrm{Cl}_{2} / \mathrm{MeOH}, 10: 0.2\right)$. ${ }^{1} \mathrm{H}$ NMR $\left[400 \mathrm{MHz}, \delta(\mathrm{ppm}), \mathrm{CDCl}_{3}\right]: 8.38(\mathrm{dd}, J=2.7,1.5 \mathrm{~Hz}, 1 \mathrm{H})$, $7.92(\mathrm{~s}, 1 \mathrm{H}), 7.81-7.75(\mathrm{~m}, 2 \mathrm{H}), 7.66$ (dddd, $J=7.7,1.5,1.0,0.5 \mathrm{~Hz}$, $1 \mathrm{H}), 7.52-7.44(\mathrm{~m}, 3 \mathrm{H}), 7.38(\mathrm{dd}, J=8.3,7.7 \mathrm{~Hz}, 1 \mathrm{H}), 7.05$ (ddd, $J=$ 8.3, 2.7, $1.0 \mathrm{~Hz}, 1 \mathrm{H}), 3.89(\mathrm{~s}, 3 \mathrm{H}) .{ }^{13} \mathrm{C}$ NMR [101 MHz, $\delta(\mathrm{ppm})$, $\left.\mathrm{CDCl}_{3}\right]: 159.7,149.1,134.7,131.9,130.0,129.5,129.2,122.3,121.8$ 118.1, 112.7, 55.4. FTIR $\left[\bar{\nu}\left(\mathrm{cm}^{-1}\right)\right]: 3060,2834,1553,1270,1025$, 769, 684. HRMS (ESI-TOF) $m / z:[\mathrm{M}+\mathrm{Na}]^{+}$calcd for $\mathrm{C}_{14} \mathrm{H}_{13} \mathrm{NO}_{2} \mathrm{Na}$ 250.0839, found 250.0857. $R_{f}: 0.06\left(\mathrm{CH}_{2} \mathrm{Cl}_{2}\right)$. Yield: $98 \%$.

(Z)-N-Phenyl-1-[3-(trifluoromethyl)phenyl]methanimine Oxide $(6 \mathrm{k}){ }^{25}$ According to general procedure $\mathrm{B}$, the reaction of 3 (trifluoromethyl)benzaldehyde $(61 \mu \mathrm{L}, 0.458 \mathrm{mmol})$ with $N$-phenylhydroxylamine $(50 \mathrm{mg}, 0.458 \mathrm{mmol})$ afforded nitrone $6 \mathrm{k}(116 \mathrm{mg}$, $0.437 \mathrm{mmol}$ ) as a white solid, after column chromatography (heptane/ AcOEt, $5: 1 \rightarrow 1: 1) .{ }^{1} \mathrm{H}$ NMR [400 MHz, $\delta(\mathrm{ppm}), \mathrm{CDCl}_{3}$ ]: $8.70(\mathrm{dq}, J$ $=2.3,1.0 \mathrm{~Hz}, 1 \mathrm{H}), 8.59$ (dddd $J=7.9,1.7,1.2,0.6 \mathrm{~Hz}, 1 \mathrm{H}), 8.01(\mathrm{~s}, 1$ $\mathrm{H}), 7.84-7.75(\mathrm{~m}, 2 \mathrm{H}), 7.72(\mathrm{dq}, J=8.5,1.1 \mathrm{~Hz}, 1 \mathrm{H}), 7.61(\mathrm{t}, J=7.9$ $\mathrm{Hz}, 1 \mathrm{H}), 7.55-7.48(\mathrm{~m}, 3 \mathrm{H}) .{ }^{13} \mathrm{C}$ NMR $\left[101 \mathrm{MHz}, \delta(\mathrm{ppm}), \mathrm{CDCl}_{3}\right]$ : $149.1,133.1,131.8$ (q, $J=1.0 \mathrm{~Hz}), 131.5,131.5,130.5,129.5,129.3$, $127.3(\mathrm{q}, J=3.7 \mathrm{~Hz}), 125.7(\mathrm{q}, J=3.9 \mathrm{~Hz}), 122.6,121.9$. FTIR $[\bar{\nu}$ $\left.\left(\mathrm{cm}^{-1}\right)\right]: 3124,1604,1330,1120,1068,770,692$. HRMS (ESI-TOF) $m / z:[\mathrm{M}+\mathrm{H}]^{+}$calcd for $\mathrm{C}_{14} \mathrm{H}_{11} \mathrm{~F}_{3} \mathrm{NO}$ 266.0787, found 266.0799. $R_{f}$ : $0.35\left(\mathrm{CH}_{2} \mathrm{Cl}_{2}\right)$. Yield: $95 \%$.

(Z)-N-Phenyl-1-(2-tolyl)methanimine Oxide (6I). ${ }^{26}$ According to general procedure $\mathrm{A}$, the reaction of 2-methylbenzaldehyde $(0.061 \mathrm{~mL}$, $0.524 \mathrm{mmol})$ with $N$-phenylhydroxylamine $(60 \mathrm{mg}, 0.551 \mathrm{mmol})$ afforded nitrone $6 \mathbf{l}$ (107 mg, $0.508 \mathrm{mmol}$ ) as a yellow-brown solid, after column chromatography $\left(\mathrm{CH}_{2} \mathrm{Cl}_{2} \rightarrow \mathrm{CH}_{2} \mathrm{Cl}_{2} / \mathrm{MeOH}, 10: 0.2\right) .{ }^{1} \mathrm{H}$ NMR $\left[400 \mathrm{MHz}, \delta(\mathrm{ppm}), \mathrm{CDCl}_{3}\right]: 9.42-9.32(\mathrm{~m}, 1 \mathrm{H}), 8.07(\mathrm{~s}, 1 \mathrm{H})$, 7.81-7.73 (m, $2 \mathrm{H}), 7.53-7.44(\mathrm{~m}, 3 \mathrm{H}), 7.39-7.33(\mathrm{~m}, 2 \mathrm{H}), 7.28-$ $7.23(\mathrm{~m}, 1 \mathrm{H}), 2.46(\mathrm{~s}, 3 \mathrm{H})$. Yield: 97\%.

(Z)-1-(2-Methoxyphenyl)-N-phenylmethanimine Oxide $(6 \mathrm{~m}){ }^{25}$ According to general procedure $\mathrm{A}$, the reaction of 2-methoxybenzaldehyde $(70 \mathrm{mg}, 0.514 \mathrm{mmol}$ ) with $\mathrm{N}$-phenylhydroxylamine $(59 \mathrm{mg}, 0.540$ $\mathrm{mmol}$ ) afforded nitrone $6 \mathrm{~m}(106 \mathrm{mg}, 0.464 \mathrm{mmol})$ as an orange-brown solid, after column chromatography $\left(\mathrm{CH}_{2} \mathrm{Cl}_{2} \rightarrow \mathrm{CH}_{2} \mathrm{Cl}_{2} / \mathrm{MeOH}\right.$, 10:0.2). ${ }^{1} \mathrm{H}$ NMR [400 MHz, $\delta$ (ppm), $\mathrm{CDCl}_{3}$ ]: 9.48 (dd, $J=7.9,1.7$ $\mathrm{Hz}, 1 \mathrm{H}), 8.40(\mathrm{~s}, 1 \mathrm{H}), 7.85-7.73(\mathrm{~m}, 2 \mathrm{H}), 7.53-7.39(\mathrm{~m}, 4 \mathrm{H})$, $7.13-7.06(\mathrm{~m}, 1 \mathrm{H}), 6.93(\mathrm{dd}, J=8.4,1.1 \mathrm{~Hz}, 1 \mathrm{H}), 3.89(\mathrm{~s}, 3 \mathrm{H}) .{ }^{13} \mathrm{C}$ NMR [101 MHz, $\delta$ (ppm), $\left.\mathrm{CDCl}_{3}\right]: 157.6,149.8,132.3,129.8,129.4$, $129.2,128.9,122.0,121.0,120.1,110.0,55.8$. FTIR $\left[\bar{\nu}\left(\mathrm{cm}^{-1}\right)\right]: 3058$, 1577, 1288, 1046, 746, 691, 611. $R_{f}: 0.07\left(\mathrm{CH}_{2} \mathrm{Cl}_{2}\right)$. Yield: $90 \%$.

(Z)-N-Phenyl-1-(thiophen-3-yl)methanimine Oxide (6n). ${ }^{27} \mathrm{Ac}-$ cording to general procedure $\mathrm{A}$, the reaction of thiophene-3carbaldehyde $(0.047 \mathrm{~mL}, 0.535 \mathrm{mmol})$ with $N$-phenylhydroxylamine (61 mg, $0.562 \mathrm{mmol}$ ) afforded nitrone $6 \mathrm{n}(109 \mathrm{mg}, 0.535 \mathrm{mmol})$ as a white-yellow solid, after column chromatography (heptane $/ \mathrm{CH}_{2} \mathrm{Cl}_{2}$, $1: 1 \rightarrow$ heptane $\left./ \mathrm{CH}_{2} \mathrm{Cl}_{2}, 0: 1\right) .{ }^{1} \mathrm{H}$ NMR $\left[400 \mathrm{MHz}, \delta(\mathrm{ppm}), \mathrm{CDCl}_{3}\right]$ : 9.16 (ddd, $J=3.1,1.2,0.7 \mathrm{~Hz}, 1 \mathrm{H}), 8.06(\mathrm{~d}, J=0.6 \mathrm{~Hz}, 1 \mathrm{H}), 7.82-$ $7.76(\mathrm{~m}, 2 \mathrm{H}), 7.52-7.46$ (m, $3 \mathrm{H}), 7.45$ (dd, $J=5.2,1.2 \mathrm{~Hz}, 1 \mathrm{H}), 7.39$ (dd, $J=5.1,3.0 \mathrm{~Hz}, 1 \mathrm{H})$. Yield: $99 \%$.

(Z)-1-(1H-Indol-2-yl)-N-phenylmethanimine Oxide (6o). According to general procedure $\mathrm{A}$, the reaction of $1 \mathrm{H}$-indole-2-carbaldehyde $(80 \mathrm{mg}, 0.551 \mathrm{mmol})$ with $\mathrm{N}$-phenylhydroxylamine $(63 \mathrm{mg}, 0.579$ $\mathrm{mmol})$ afforded nitrone $60(130 \mathrm{mg}, 0.551 \mathrm{mmol})$ as a yellow-brown solid. ${ }^{1} \mathrm{H}$ NMR [ $500 \mathrm{MHz}, \delta(\mathrm{ppm}), \mathrm{CDCl}_{3}$ ]: $11.69(\mathrm{~s}, 1 \mathrm{H}), 8.14(\mathrm{~s}, 1$ H), 7.87-7.80 (m, $2 \mathrm{H}), 7.67(\mathrm{dd}, J=8.1,1.0 \mathrm{~Hz}, 1 \mathrm{H}), 7.55-7.45(\mathrm{~m}$, $4 \mathrm{H}$ ), 7.32 (ddd, $J=8.2,6.9,1.1 \mathrm{~Hz}, 1 \mathrm{H}), 7.16$ (ddd, $J=8.0,6.9,1.0$ $\mathrm{Hz}, 1 \mathrm{H}), 6.94(\mathrm{~s}, 1 \mathrm{H}) .{ }^{13} \mathrm{C}$ NMR $\left[126 \mathrm{MHz}, \delta(\mathrm{ppm}), \mathrm{CDCl}_{3}\right]: 147.4$, $136.0,130.2,129.9,129.4,127.6,126.8,125.2,121.3,120.8,112.3$, 108.6. FTIR $\left[\bar{\nu}\left(\mathrm{cm}^{-1}\right)\right]: 3315,3067,1593,1502,1345,1234,818,733$. HRMS (ESI-TOF) $m / z:[\mathrm{M}+\mathrm{Na}]^{+}$calcd for $\mathrm{C}_{15} \mathrm{H}_{12} \mathrm{~N}_{2} \mathrm{ONa} 259.0842$, found 259.0858. $R_{f}: 0.13\left(\mathrm{CH}_{2} \mathrm{Cl}_{2}\right)$. Yield: $99 \%$.

(Z)-N-Phenyl-1-(1H-pyrrol-2-yl)methanimine Oxide (6p). According to general procedure $\mathrm{A}$, the reaction of $1 \mathrm{H}$-pyrrole-2-carbaldehyde (52 mg, $0.547 \mathrm{mmol}$ ) with $\mathrm{N}$-phenylhydroxylamine $(60 \mathrm{mg}, 0.550$ $\mathrm{mmol})$ afforded nitrone $6 \mathrm{p}(62 \mathrm{mg}, 0.333 \mathrm{mmol})$ as a dark brown solid, after column chromatography $\left(\mathrm{CH}_{2} \mathrm{Cl}_{2} \rightarrow \mathrm{CH}_{2} \mathrm{Cl}_{2} / \mathrm{MeOH}, 10: 0.1\right)$. ${ }^{1} \mathrm{H}$ NMR $\left[500 \mathrm{MHz}, \delta(\mathrm{ppm}), \mathrm{CDCl}_{3}\right]: 12.21(\mathrm{~s}, 1 \mathrm{H}), 7.95(\mathrm{~s}, 1 \mathrm{H})$, $7.81-7.73(\mathrm{~m}, 2 \mathrm{H}), 7.52-7.38(\mathrm{~m}, 3 \mathrm{H}), 7.07(\mathrm{td}, J=2.7,1.3 \mathrm{~Hz}, 1$ $\mathrm{H}), 6.70(\mathrm{dt}, J=3.6,1.6 \mathrm{~Hz}, 1 \mathrm{H}), 6.40(\mathrm{dt}, J=3.9,2.5 \mathrm{~Hz}, 1 \mathrm{H}) .{ }^{13} \mathrm{C}$ NMR $\left[126 \mathrm{MHz}, \delta\right.$ (ppm), $\left.\mathrm{CDCl}_{3}\right]: 147.1,129.5,129.3,126.1,125.3$, 121.3, 121.0, 115.9, 111.3. FTIR $\left[\bar{\nu}\left(\mathrm{cm}^{-1}\right)\right]: 3317,3062,1580,1403$, 1294, 1074, 1045, 740, 686. HRMS (ESI-TOF) $\mathrm{m} / z:[\mathrm{M}+\mathrm{H}]^{+}$calcd for $\mathrm{C}_{11} \mathrm{H}_{11} \mathrm{~N}_{2} \mathrm{O}$ 187.0866, found 187.0885. $R_{f}$ : 0.45 (heptane/AcOEt, $1: 1)$. Yield: $61 \%$.

(Z)-1-(Furan-2-yl)-N-phenylmethanimine Oxide (6q). ${ }^{28}$ According to general procedure $\mathrm{A}$, the reaction of furan-2-carbaldehyde ( 0.043 $\mathrm{mL}, 0.520 \mathrm{mmol}$ ) with $N$-phenylhydroxylamine $(60 \mathrm{mg}, 0.546 \mathrm{mmol})$ afforded nitrone $\mathbf{6 q}(97 \mathrm{mg}, 0.520 \mathrm{mmol})$ as a light brown solid, after column chromatography $\left(\mathrm{CH}_{2} \mathrm{Cl}_{2} \rightarrow \mathrm{CH}_{2} \mathrm{Cl}_{2} / \mathrm{MeOH}, 10: 0.2\right) .{ }^{1} \mathrm{H}$ NMR [400 MHz, $\left.\delta(\mathrm{ppm}), \mathrm{CDCl}_{3}\right]: 8.16(\mathrm{~s}, 1 \mathrm{H}), 8.01(\mathrm{dt}, J=3.5,0.7$ $\mathrm{Hz}, 1 \mathrm{H}), 7.83-7.77(\mathrm{~m}, 2 \mathrm{H}), 7.58(\mathrm{dd}, J=1.8,0.7 \mathrm{~Hz}, 1 \mathrm{H}), 7.52-$ $7.44(\mathrm{~m}, 3 \mathrm{H}), 6.65$ (dd, $J=3.6,1.8 \mathrm{~Hz}, 1 \mathrm{H})$. Yield: 99\%.

(Z)-2-(Benzylamino)-2-oxo-N-phenylethan-1-imine Oxide (6r). According to general procedure $\mathrm{A}$, the reaction of $\mathrm{N}$-benzyl-2oxoacetamide (56 mg, $0.343 \mathrm{mmol}$ ) with $\mathrm{N}$-phenylhydroxylamine $(26 \mathrm{mg}, 0.240 \mathrm{mmol})$ afforded nitrone $6 \mathrm{r}(34.5 \mathrm{mg}, 0.135 \mathrm{mmol})$ as a white solid. ${ }^{1} \mathrm{H}$ NMR [400 MHz, $\delta$ (ppm), $\mathrm{CDCl}_{3}$ ]: 10.39 (s, $\left.1 \mathrm{H}\right)$, $7.72-7.67(\mathrm{~m}, 2 \mathrm{H}), 7.66(\mathrm{~s}, 1 \mathrm{H}), 7.58-7.46(\mathrm{~m}, 3 \mathrm{H}), 7.40-7.27(\mathrm{~m}$, $5 \mathrm{H}), 4.64(\mathrm{~d}, J=5.9 \mathrm{~Hz}, 2 \mathrm{H}) .{ }^{13} \mathrm{C}$ NMR $\left[101 \mathrm{MHz}, \delta(\mathrm{ppm}), \mathrm{CDCl}_{3}\right]$ : $160.8,147.5,137.8,131.7,130.3,129.7,128.9,128.0,127.7,121.6,43.3$. FTIR $\left[\bar{\nu}\left(\mathrm{cm}^{-1}\right)\right]: 3255,3121,2945,1634,1516,1059,768,751,683$. HRMS (ESI-TOF) $m / z:\left[\mathrm{M}+\mathrm{Na}^{+}\right.$calcd for $\mathrm{C}_{15} \mathrm{H}_{14} \mathrm{~N}_{2} \mathrm{O}_{2} \mathrm{Na}$ 277.0948, found 277.0956. $R_{f}$ : $0.68\left(\mathrm{CH}_{2} \mathrm{Cl}_{2} / \mathrm{MeOH}, 10: 0.2\right)$. Yield: $39 \%$. 
General Procedure for the Synthesis of Isoxazolidines $4 \mathrm{a}, \mathbf{4 b}$, $\mathbf{4 d}-\mathbf{r}$. The solution containing alkene 2 in distilled THF ( 3 equiv, 1.48 $\mathrm{M})$ at $0{ }^{\circ} \mathrm{C}$ was added to a PTFE tube containing the corresponding nitrone 6 ( 1 equiv) and galvinoxyl ( 0.03 equiv). The tube was filled up with distilled THF, closed and brought to $15 \mathrm{kbar}$ at $50{ }^{\circ} \mathrm{C}$ for $16 \mathrm{~h}$. Then, the reaction mixture was filtered off, and the solvent was removed under vacuo. The crude mixture was purified by column chromatography using the indicated eluent to afford isoxazolidines $4 a$, $\mathbf{4 b}$, and $\mathbf{4 d}-\mathbf{r}$.

2,3-Diphenyl-5-[(trifluoromethyl)sulfanyl]isoxazolidine (4a). According to the general procedure, the reaction of nitrone $6 \mathrm{a}(26 \mathrm{mg}$, $0.119 \mathrm{mmol}$ ) and alkene 2 in a $1 \mathrm{~mL}$ PTFE high-pressure tube afforded cis-4a $(24.9 \mathrm{mg}, 0.77 \mathrm{mmol}, 65 \%)$ as a white solid and a mixture of cisand trans-4a and regioisomers $7 \mathbf{a}(12.6 \mathrm{mg}, 0.039 \mathrm{mmol}, 33 \%)$ as a yellow oil after column chromatography (heptane $/ \mathrm{CH}_{2} \mathrm{Cl}_{2}, 9: 1$ ). Total yield: $98 \%$.

rac-(3R,5R)-2,3-Diphenyl-5-[(trifluoromethyl)sulfanyl]isoxazolidine (cis-4a). ${ }^{1} \mathrm{H}$ NMR $\left[500 \mathrm{MHz}, \delta(\mathrm{ppm}), \mathrm{CDCl}_{3}\right]: 7.52-$ 7.47 (m, $2 \mathrm{H}), 7.43-7.37$ (m, 2 H), 7.35-7.30 (m, $1 \mathrm{H}), 7.24-7.18$ (m, $2 \mathrm{H}), 7.04-6.99,(\mathrm{~m}, 1 \mathrm{H}), 6.99-6.95(\mathrm{~m}, 2 \mathrm{H}), 6.01(\mathrm{dd}, J=8.1,4.4$ $\mathrm{Hz}, 1 \mathrm{H}), 4.41$ (dd, $J=9.0,7.1 \mathrm{~Hz}, 1 \mathrm{H}), 3.41$ (dddq, $J=13.6,9.0,8.1$, $0.9 \mathrm{~Hz}, 1 \mathrm{H}), 2.44-2.31(\mathrm{~m}, 1 \mathrm{H}) .{ }^{13} \mathrm{C}$ NMR [126 MHz, $\delta(\mathrm{ppm})$, $\left.\mathrm{CDCl}_{3}\right]: 148.8,139.8,130.2(\mathrm{q}, J=307.7 \mathrm{~Hz}), 129.3,128.8,128.3$, $127.2,123.8,117.6,80.5(\mathrm{q}, J=2.5 \mathrm{~Hz}), 68.3,47.2$. FTIR $\left[\bar{\nu}\left(\mathrm{cm}^{-1}\right)\right]$ : 3070, 2963, 1598, 1489, 1258, 1114, 1031, 966, 928, 752, 700. HRMS (EI) $\mathrm{m} / z$ : $\left[\mathrm{M}^{\bullet}\right]$ calcd for $\mathrm{C}_{16} \mathrm{H}_{14} \mathrm{~F}_{3} \mathrm{NOS} 325.0748$, found 325.0765 . $R_{f}$ : 0.20 (heptane $/ \mathrm{CH}_{2} \mathrm{Cl}_{2}, 9: 1$ ).

rac-(3R,5S)-2,3-Diphenyl-5-[(trifluoromethyl)sulfanyl]isoxazolidine (trans-4a). ${ }^{1} \mathrm{H}$ NMR $\left[500 \mathrm{MHz}, \delta(\mathrm{ppm}), \mathrm{CDCl}_{3}\right]$ : $7.50-7.45(\mathrm{~m}, 2 \mathrm{H}), 7.41-7.36(\mathrm{~m}, 2 \mathrm{H}), 7.35-7.30(1 \mathrm{H}), 7.25-7.18$ (m, $2 \mathrm{H}), 7.04-6.98(\mathrm{~m}, 2 \mathrm{H}), 6.98-6.93(\mathrm{~m}, 1 \mathrm{H}), 6.07(\mathrm{dd}, J=6.8$, $3.8 \mathrm{~Hz}, 1 \mathrm{H}), 4.90(\mathrm{t}, J=7.3 \mathrm{~Hz}, 1 \mathrm{H}), 3.02-2.92(\mathrm{~m}, 1 \mathrm{H}), 2.88(\mathrm{ddd}, J$ $=13.3,7.3,3.8 \mathrm{~Hz}, 1 \mathrm{H}) .{ }^{13} \mathrm{C}$ NMR $\left[126 \mathrm{MHz}, \delta(\mathrm{ppm}), \mathrm{CDCl}_{3}\right]$ : $151.0,139.9,129.9(\mathrm{q}, J=307.8 \mathrm{~Hz}), 129.2,128.8,128.2,126.7,122.4$, 115.2, $82.3(\mathrm{q}, J=2.0 \mathrm{~Hz}), 68.1,46.3$. FTIR $\left[\bar{\nu}\left(\mathrm{cm}^{-1}\right)\right]: 3065,1598$, 1489, 1206, 1108, 1049, 842, 796, 665. HRMS (EI) $\mathrm{m} / \mathrm{z}:\left[\mathrm{M}^{+\bullet}\right]$ calcd for $\mathrm{C}_{16} \mathrm{H}_{14} \mathrm{~F}_{3} \mathrm{NOS} 325.0748$, found 325.0750. $R_{f}: 0.11$ (heptane/ $\left.\mathrm{CH}_{2} \mathrm{Cl}_{2}, 9: 1\right)$.

3-(4-Fluorophenyl)-2-phenyl-5-[(trifluoromethyl)sulfanyl]isoxazolidine (4b). According to the general procedure, the reaction of nitrone $6 \mathrm{~b}(27 \mathrm{mg}, 0.125 \mathrm{mmol})$ and alkene 2 in a $1 \mathrm{~mL}$ PTFE highpressure tube afforded cis- $4 \mathrm{~b}(27.6 \mathrm{mg}, 0.080 \mathrm{mmol}, 64 \%)$ as a white solid, a mixture of cis- and trans- $4 \mathbf{b}$ and regioisomers $7 \mathbf{b}(13.1 \mathrm{mg}, 0.038$ $\mathrm{mmol}, 27 \%)$ as a yellow oil and trans- $4 \mathbf{b}(3.5 \mathrm{mg}, 0.010 \mathrm{mmol}, 8 \%)$ as a pale yellow oil, after column chromatography (heptane $/ \mathrm{CH}_{2} \mathrm{Cl}_{2}, 9: 1$ ). Total yield: $99 \%$.

rac-(3R,5R)-3-(4-Fluorophenyl)-2-phenyl-5-[(trifluoromethyl)sulfanyl]isoxazolidine (cis-4b). ${ }^{1} \mathrm{H}$ NMR $[500 \mathrm{MHz}, \delta$ (ppm), $\mathrm{CDCl}_{3}$ ]: 7.49-7.43 (m, $\left.2 \mathrm{H}\right), 7.25-7.19(\mathrm{~m}, 2 \mathrm{H}), 7.11-7.05(\mathrm{~m}, 2$ $\mathrm{H}), 7.05-7.01(\mathrm{~m}, 1 \mathrm{H}), 6.99-6.93(\mathrm{~m}, 2 \mathrm{H}), 6.01(\mathrm{dd}, J=8.1,4.3 \mathrm{~Hz}$, $1 \mathrm{H}), 4.40$ (dd, $J=9.0,7.0 \mathrm{~Hz}, 1 \mathrm{H}), 3.39$ (dddq, $J=13.7,9.0,8.1,0.9$ $\mathrm{Hz}, 1 \mathrm{H}), 2.39-2.28(\mathrm{~m}, 1 \mathrm{H}) .{ }^{13} \mathrm{C}$ NMR [126 MHz, $\left.\delta(\mathrm{ppm}), \mathrm{CDCl}_{3}\right]$ : $162.6(\mathrm{~d}, J=247.0 \mathrm{~Hz}), 148.6,135.4(\mathrm{~d}, J=3.1 \mathrm{~Hz}), 130.1(\mathrm{q}, J=307.8$ $\mathrm{Hz}), 128.92,128.91(\mathrm{~d}, J=7.2 \mathrm{~Hz}), 124.0,117.7,116.2(\mathrm{~d}, J=21.6 \mathrm{~Hz})$, $80.5(\mathrm{q}, J=2.3 \mathrm{~Hz}), 67.7,47.1 .{ }^{19} \mathrm{~F} \mathrm{NMR}\left[471 \mathrm{MHz}, \delta(\mathrm{ppm}), \mathrm{CDCl}_{3}\right]$ : -39.9. FTIR $\left[\bar{\nu}\left(\mathrm{cm}^{-1}\right)\right]: 3071,1597,1455,1256,1226,1110,1029$, 905, 804, 754. HRMS (EI) $m / z:\left[\mathrm{M}^{+\bullet}\right]$ calcd for $\mathrm{C}_{16} \mathrm{H}_{13} \mathrm{~F}_{4} \mathrm{NOS}$ 343.0654, found 343.0650. $R_{f}: 0.26$ (heptane $/ \mathrm{CH}_{2} \mathrm{Cl}_{2}, 17: 3$ ).

rac-(3R,5S)-3-(4-Fluorophenyl)-2-phenyl-5-[(trifluoromethyl]sulfanyl]isoxazolidine (trans-4b). ${ }^{1} \mathrm{H}$ NMR $[500 \mathrm{MHz}, \delta(\mathrm{ppm})$, $\mathrm{CDCl}_{3}$ ]: 7.44-7.40 (m, $\left.2 \mathrm{H}\right), 7.25-7.20(\mathrm{~m}, 2 \mathrm{H}), 7.10-7.04(\mathrm{~m}, 2$ $\mathrm{H}), 6.99-6.92(\mathrm{~m}, 3 \mathrm{H}), 6.05(\mathrm{dd}, J=6.8,3.9 \mathrm{~Hz}, 1 \mathrm{H}), 4.88(\mathrm{t}, J=7.2$ $\mathrm{Hz}, 1 \mathrm{H}), 2.98-2.90(\mathrm{~m}, 1 \mathrm{H}), 2.87(\mathrm{ddd}, J=13.3,7.3,3.9 \mathrm{~Hz}, 1 \mathrm{H})$. ${ }^{13} \mathrm{C}$ NMR $\left[126 \mathrm{MHz}, \delta(\mathrm{ppm}), \mathrm{CDCl}_{3}\right]: 162.3(\mathrm{~d}, J=242.3 \mathrm{~Hz}), 150.7$, 135.6, 128.9, 128.4 (d, $J=8.3 \mathrm{~Hz}), 122.6,116.2(\mathrm{~d}, J=21.7 \mathrm{~Hz}), 115.3$, 81.2, 67.4, 46.2. The carbon signal of $\mathrm{SCF}_{3}$ was not observed. ${ }^{19} \mathrm{~F}$ NMR [471 MHz, $\left.\delta(\mathrm{ppm}), \mathrm{CDCl}_{3}\right]:-39.4$. FTIR $\left[\bar{\nu}\left(\mathrm{cm}^{-1}\right)\right]: 3071,1686$, $1598,1154,1052,937,693$. HRMS (EI) $\mathrm{m} / z:\left[\mathrm{M}^{\bullet}\right]$ calcd for $\mathrm{C}_{16} \mathrm{H}_{13} \mathrm{~F}_{4} \mathrm{NOS}$ 343.0654, found 343.0676. $R_{f}$ : 0.21 (heptane $/ \mathrm{CH}_{2} \mathrm{Cl}_{2}$, $17: 3)$.
2-Phenyl-3-(4-tolyl)-5-[(trifluoromethyl)sulfanyl]isoxazolidine (4c). The solution containing alkene 2 (3.0 equiv) in distilled THF $(500 \mu \mathrm{L})$ at $0{ }^{\circ} \mathrm{C}$ was added to a PTFE tube containing nitrone $6 \mathrm{c}(1.0$ equiv). The tube was filled up with distilled THF, closed and brought to $15 \mathrm{kbar}$ at $50{ }^{\circ} \mathrm{C}$ for $16 \mathrm{~h}$. Then, the reaction mixture was filtered off and the solvent was removed under vacuo. The crude mixture was purified by column chromatography (heptane/ $\mathrm{CH}_{2} \mathrm{Cl}_{2}, 4: 1$ ) to afford isoxazolidine cis-4c $(17.1 \mathrm{mg}, 0.050 \mathrm{mmol}, 50 \%)$ as white solid and trans-4c ( $12.1 \mathrm{mg}, 0.36 \mathrm{mmol}, 35 \%)$ as a brown oil, contaminated with regioisomers $7 \mathrm{c}$ and azoxybenzene 8 . Total yield: $85 \%$.

rac-(3R,5R)-2-Phenyl-3-(4-tolyl)-5-[(trifluoromethyl)sulfanyl]isoxazolidine (cis-4c). ${ }^{1} \mathrm{H}$ NMR [500 $\left.\mathrm{MHz}, \delta(\mathrm{ppm}), \mathrm{CDCl}_{3}\right]$ : 7.40$7.34(\mathrm{~m}, 2 \mathrm{H}), 7.23-7.16(\mathrm{~m}, 4 \mathrm{H}), 7.03-6.95(\mathrm{~m}, 3 \mathrm{H}), 6.00(\mathrm{dd}, J=$ 8.1, $4.3 \mathrm{~Hz}, 1 \mathrm{H}), 4.36(\mathrm{dd}, J=9.0,7.2 \mathrm{~Hz}, 1 \mathrm{H}), 3.38(\mathrm{dddq}, J=13.7$, 9.0, 8.1, 0.9 Hz, 1 H), 2.39-2.32 (m, $1 \mathrm{H}), 2.36(\mathrm{~s}, 3 \mathrm{H}) .{ }^{13} \mathrm{C}$ NMR $\left[126 \mathrm{MHz}, \delta(\mathrm{ppm}), \mathrm{CDCl}_{3}\right]: 148.9,138.1,136.6,130.2(\mathrm{q}, J=307.7$ $\mathrm{Hz}), 130.0,128.8,127.1,123.7,117.6,80.5$ (q, $J=2.2 \mathrm{~Hz}), 68.1,47.3$, 21.3. ${ }^{19} \mathrm{~F}$ NMR $\left[471 \mathrm{MHz}, \delta(\mathrm{ppm}), \mathrm{CDCl}_{3}\right]:-39.9$. FTIR $\left[\bar{\nu}\left(\mathrm{cm}^{-1}\right)\right]$ : 3056, 2962, 1597, 1489, 1257, 1107, 752, 691. HRMS (EI) $\mathrm{m} / z:\left[\mathrm{M}^{+\bullet}\right]$ calcd for $\mathrm{C}_{17} \mathrm{H}_{16} \mathrm{~F}_{3} \mathrm{NOS} 339.0905$, found 339.0890. Rf: 0.21 (heptane/ $\left.\mathrm{CH}_{2} \mathrm{Cl}_{2}, 4: 1\right)$.

rac-(3R,5S)-2-Phenyl-3-(4-tolyl)-5-[(trifluoromethyl)sulfanyl]isoxazolidine (trans-4c). ${ }^{1} \mathrm{H}$ NMR $\left[500 \mathrm{MHz}, \delta\right.$ (ppm), $\left.\mathrm{CDCl}_{3}\right]$ : 7.36-7.33 (m, $2 \mathrm{H}), 7.25-7.16(\mathrm{~m}, 4 \mathrm{H}), 7.02-6.98(\mathrm{~m}, 2 \mathrm{H}), 6.97-$ $6.92(\mathrm{~m}, 1 \mathrm{H}), 6.06(\mathrm{dd}, J=6.8,3.8 \mathrm{~Hz}, 1 \mathrm{H}), 4.85(\mathrm{t}, J=7.2 \mathrm{~Hz}, 1 \mathrm{H})$, 2.95 (dddq, $J=13.2,7.6,6.8,0.9 \mathrm{~Hz}, 1 \mathrm{H}$ ), 2.85 (ddd, $J=13.2,7.2,3.8$ $\mathrm{Hz}, 1 \mathrm{H}), 2.36(\mathrm{~s}, 3 \mathrm{H}) .{ }^{13} \mathrm{C}$ NMR [126 MHz, $\left.\delta(\mathrm{ppm}), \mathrm{CDCl}_{3}\right]: 151.0$, 137.9, 136.9, 130.1 (q, $J=307.7 \mathrm{~Hz}), 129.8,128.8,126.6,122.2,115.2$, $82.3(\mathrm{q}, J=2.2 \mathrm{~Hz}), 67.9,46.4,21.3 .{ }^{19} \mathrm{~F}$ NMR [ $471 \mathrm{MHz}, \delta(\mathrm{ppm})$, $\left.\mathrm{CDCl}_{3}\right]:-39.3$. FTIR $\left[\bar{\nu}\left(\mathrm{cm}^{-1}\right)\right]: 3063,2925,1599,1487,1152,1053$, 756, 686. HRMS (EI) $m / z:\left[\mathrm{M}^{\bullet}\right]$ calcd for $\mathrm{C}_{17} \mathrm{H}_{16} \mathrm{~F}_{3} \mathrm{NOS} 339.0905$, found 339.0924. $R_{f}: 0.13$ (heptane/ $\mathrm{CH}_{2} \mathrm{Cl}_{2}, 4: 1$ ).

2-Phenyl-3-[4-(trifluoromethyl)phenyl]-5-[(trifluoromethyl)sulfanyl]isoxazolidine (4d). According to the general procedure, the reaction of nitrone $6 \mathrm{~d}(50 \mathrm{mg}, 0.190 \mathrm{mmol})$ and alkene 2 in a $1.5 \mathrm{~mL}$ PTFE high-pressure tube, containing approximately $300 \mu \mathrm{L}$ of glass beads, afforded a mixture of isoxazolidines cis- and trans-4d and regioisomers $7 \mathrm{~d}(53.1 \mathrm{mg}, 0.137 \mathrm{mmol}, 73 \%)$ as a pale yellow solid oil, after column chromatography (heptane $/ \mathrm{CH}_{2} \mathrm{Cl}_{2}, 17: 3$ ). FTIR $[\bar{\nu}$ $\left.\left(\mathrm{cm}^{-1}\right)\right]: 1597,1490,1325,1104,1051,850,754,692$. HRMS (EI) $\mathrm{m} /$ $z:\left[\mathrm{M}^{+\bullet}\right]$ calcd for $\mathrm{C}_{17} \mathrm{H}_{13} \mathrm{~F}_{6} \mathrm{NOS} 393.0622$, found 393.0624. $R_{f}: 0.20$ (heptane $\left./ \mathrm{CH}_{2} \mathrm{Cl}_{2}, 17: 3\right)$. Total yield: $73 \%$.

rac-(3R,5R)-2-Phenyl-3-[4-(trifluoromethyl)phenyl]-5[(trifluoromethyl)sulfanyl]isoxazolidine (cis-4d). ${ }^{1} \mathrm{H}$ NMR $[500 \mathrm{MHz}$, $\delta(\mathrm{ppm}), \mathrm{CDCl}_{3}$ ]: 7.72-7.55 (m, $\left.4 \mathrm{H}\right), 7.25-7.18(\mathrm{~m}, 2 \mathrm{H}), 7.05-7.00$ $(\mathrm{m}, 1 \mathrm{H}), 6.97-6.93(\mathrm{~m}, 2 \mathrm{H}), 6.00(\mathrm{dd}, J=8.0,4.3 \mathrm{~Hz}, 1 \mathrm{H}), 4.50(\mathrm{dd}$, $J=9.1,6.7,1 \mathrm{H}), 3.43$ (dddq, $J=13.7,9.1,8.0,0.9,1 \mathrm{H}), 2.33$ (ddd, $J=$ 13.7, 6.7, $4.3 \mathrm{~Hz}, 1 \mathrm{H}) .{ }^{13} \mathrm{C}$ NMR $\left[126 \mathrm{MHz}, \delta(\mathrm{ppm}), \mathrm{CDCl}_{3}\right]$ : 148.4, $144.1,130.6(\mathrm{q}, J=32.6 \mathrm{~Hz}), 130.0(\mathrm{q}, J=307.8 \mathrm{~Hz}), 129.0,127.4$, $126.3(\mathrm{q}, J=3.8 \mathrm{~Hz}), 124.09(\mathrm{q}, J=272.1 \mathrm{~Hz}), 124.05,117.4,80.4(\mathrm{q}, J$ $=2.2 \mathrm{~Hz}), 67.5,46.9$.

rac-(3R,5S)-2-Phenyl-3-[4-(trifluoromethyl)phenyl]-5[(trifluoromethyl)sulfanyl]isoxazolidine (trans-4d). ${ }^{1} \mathrm{H}$ NMR [500 MHz, $\delta(\mathrm{ppm}), \mathrm{CDCl}_{3}$ ]: 7.70-7.56 (m, $\left.4 \mathrm{H}\right), 7.27-7.18(\mathrm{~m}, 2 \mathrm{H})$, $7.00-6.96(\mathrm{~m}, 3 \mathrm{H}), 6.04(\mathrm{dd}, J=6.3,4.6 \mathrm{~Hz}, 1 \mathrm{H}), 4.97(\mathrm{t}, J=7.1 \mathrm{~Hz}$, $1 \mathrm{H}), 3.01-2.83(\mathrm{~m}, 2 \mathrm{H}) .{ }^{13} \mathrm{C}$ NMR [126 MHz, $\left.\delta(\mathrm{ppm}), \mathrm{CDCl}_{3}\right]$ : $150.5,144.1,130.5(\mathrm{q}, J=32.5 \mathrm{~Hz}), 130.0(\mathrm{q}, J=307.8 \mathrm{~Hz}), 129.0$, 127.0, $126.2(\mathrm{q}, J=3.9 \mathrm{~Hz}), 122.7,115.1,82.2(\mathrm{q}, J=2.1 \mathrm{~Hz}), 67.5$, 45.9.

3-[4-(Pentafluoro- $\lambda^{6}$-sulfanyl)phenyl]-2-phenyl-5-[(trifluoromethyl)sulfanyl]isoxazolidine (4e). According to the general procedure, the reaction of nitrone $6 \mathrm{e}(37.8 \mathrm{mg}, 0.143 \mathrm{mmol})$ and alkene 2 in a $1 \mathrm{~mL}$ PTFE high-pressure tube afforded a mixture of isoxazolidines cis- and trans-4e and regioisomers $7 \mathrm{e}(41 \mathrm{mg}, 0.091 \mathrm{mmol}, 72 \%)$ as a white solid, after column chromatography (heptane $/ \mathrm{CH}_{2} \mathrm{Cl}_{2}, 17: 3$ ). FTIR $\left[\bar{\nu}\left(\mathrm{cm}^{-1}\right)\right]: 2963,1670,1585,1261,1120,1104,817,753,584$. HRMS (EI) $m / z:\left[\mathrm{M}^{\bullet}\right]$ calcd for $\mathrm{C}_{16} \mathrm{H}_{13} \mathrm{~F}_{8} \mathrm{NOS}_{2}$ 451.0311, found 451.0318. $R_{f} 0.21$ (heptane $/ \mathrm{CH}_{2} \mathrm{Cl}_{2}, 17: 3$ ). Total yield: $72 \%$.

rac-(3R,5R)-3-[4-(Pentafluoro- $\lambda^{6}$-sulfanyl)phenyl]-2-phenyl-5[(trifluoromethyl)sulfanyl]isoxazolidine (cis-4e). ${ }^{1} \mathrm{H}$ NMR $[500 \mathrm{MHz}$, $\delta(\mathrm{ppm}), \mathrm{CDCl}_{3}$ ]: 7.85-7.72 (m, $\left.2 \mathrm{H}\right), 7.64-7.55$ (m, $\left.2 \mathrm{H}\right), 7.30-7.21$ 
(m, $2 \mathrm{H}), 7.09-7.02(\mathrm{~m}, 1 \mathrm{H}), 7.02-6.92(\mathrm{~m}, 2 \mathrm{H}), 6.00(\mathrm{dd}, J=8.0$, $4.3 \mathrm{~Hz}, 1 \mathrm{H}), 4.52(\mathrm{dd}, J=9.2,6.6,1 \mathrm{H}), 3.43(\mathrm{dt}, J=13.7,8.6,1 \mathrm{H})$, 2.32 (ddd, $J=13.7,6.6,4.3 \mathrm{~Hz}, 1 \mathrm{H}) .{ }^{13} \mathrm{C}$ NMR $[126 \mathrm{MHz}, \delta(\mathrm{ppm})$, $\mathrm{CDCl}_{3}$ ]: $153.7-153.4(\mathrm{~m}), 148.3,144.0,130.0(\mathrm{q}, J=307.9 \mathrm{~Hz}), 129.1$, 127.3, 127.0, 124.2, 117.3, $80.4(\mathrm{q}, J=2.3 \mathrm{~Hz}), 67.1,46.7$.

rac-(3R,5S)-3-[4-(Pentafluoro- $\lambda^{6}$-sulfanyl)phenyl]-2-phenyl-5[(trifluoromethyl)sulfanyl]isoxazolidine (trans-4e). ${ }^{1} \mathrm{H}$ NMR [500 $\left.\mathrm{MHz}, \delta(\mathrm{ppm}), \mathrm{CDCl}_{3}\right]$ : 7.80-7.74 (m, $\left.2 \mathrm{H}\right), 7.60-7.56(\mathrm{~m}, 2 \mathrm{H})$, $7.28-7.21(\mathrm{~m}, 2 \mathrm{H}), 7.01-6.97(\mathrm{~m}, 3 \mathrm{H}), 6.03(\mathrm{dd}, J=5.9,5.0,1 \mathrm{H})$, $4.97(\mathrm{t}, J=7.1 \mathrm{~Hz}, 1 \mathrm{H}), 3.01-2.80(\mathrm{~m}, 2 \mathrm{H}) .{ }^{13} \mathrm{C}$ NMR $[126 \mathrm{MHz}, \delta$ (ppm), $\mathrm{CDCl}_{3}$ ]: 153.7-153.4 (m), 150.4, 143.9, 130.0 (q, $J=307.9$ $\mathrm{Hz}), 129.0,127.1,127.0,122.8,115.1,82.2(\mathrm{q}, J=2.2 \mathrm{~Hz}), 67.1,45.8$. 3-(4-Methoxyphenyl)-2-phenyl-5-[(trifluoromethyl)sulfanyl]isoxazolidine (4f). According to the general procedure, the reaction of nitrone $6 \mathrm{f}(54 \mathrm{mg}, 0.238 \mathrm{mmol})$ and alkene 2 in a $1.5 \mathrm{~mL}$ PTFE highpressure tube afforded isoxazolidine cis- $4 \mathrm{f}(44.3 \mathrm{mg}, 0.125 \mathrm{mmol}, 53 \%)$, a mixture of isoxazolidines cis- and trans- $4 \mathrm{f}(27.3 \mathrm{mg}, 0.077 \mathrm{mmol}, 32 \%)$ and isoxazolidine trans- $\mathbf{4 f}$ mixed with cis- $4 \mathbf{f}$ and regioisomers $7 \mathbf{f}(12.4$ $\mathrm{mg}, 0.035 \mathrm{mmol}, 15 \%$ ) as yellow oils, after column chromatography (heptane $/ \mathrm{CH}_{2} \mathrm{Cl}_{2}, 7: 3$ ). Total yield: $99 \%$.

rac-(3R,5R)-3-(4-Methoxyphenyl)-2-phenyl-5-[(trifluoromethyl)sulfanyl]isoxazolidine (cis-4f). ${ }^{1} \mathrm{H}$ NMR [ $\left.500 \mathrm{MHz}, \delta(\mathrm{ppm}), \mathrm{CDCl}_{3}\right]$ : 7.43-7.35 (m, $2 \mathrm{H}), 7.23-7.18$ (m, $2 \mathrm{H}), 7.04-6.99$ (m, $1 \mathrm{H}), 6.99-$ $6.95(\mathrm{~m}, 2 \mathrm{H}), 6.94-6.90(\mathrm{~m}, 2 \mathrm{H}), 6.01(\mathrm{dd}, J=8.1,4.3 \mathrm{~Hz}, 1 \mathrm{H}), 4.34$ $(\mathrm{dd}, J=8.9,7.3 \mathrm{~Hz}, 1 \mathrm{H}), 3.82(\mathrm{~s}, 3 \mathrm{H}), 3.37$ (dddq, $J=13.8,8.9,8.2$, $0.9 \mathrm{~Hz}, 1 \mathrm{H}), 2.46-2.25(\mathrm{~m}, 1 \mathrm{H}) .{ }^{13} \mathrm{C}$ NMR $[126 \mathrm{MHz}, \delta(\mathrm{ppm})$, $\mathrm{CDCl}_{3}$ ]: 159.6, 148.8, 131.4, $130.2(\mathrm{q}, J=307.8 \mathrm{~Hz}), 128.8,128.4$, $123.8,117.8,114.6,80.5(\mathrm{q}, J=2.2 \mathrm{~Hz}), 68.0,55.4,47.2 .{ }^{19} \mathrm{~F}$ NMR $[471$ $\left.\mathrm{MHz}, \delta(\mathrm{ppm}), \mathrm{CDCl}_{3}\right]:-39.9$. FTIR $\left[\bar{\nu}\left(\mathrm{cm}^{-1}\right)\right]: 3003,1614,1515$, $1298,1103,1031,816,773$. HRMS (EI) $\mathrm{m} / z:\left[\mathrm{M}^{+\bullet}\right]$ calcd for $\mathrm{C}_{17} \mathrm{H}_{16} \mathrm{~F}_{3} \mathrm{NO}_{2} \mathrm{~S}$ 355.0854, found 355.0861. $R_{f} 0.15$ (heptane $/ \mathrm{CH}_{2} \mathrm{Cl}_{2}$, $4: 1)$.

rac-(3R,5S)-3-(4-Methoxyphenyl)-2-phenyl-5-[(trifluoromethyl)sulfanyl]isoxazolidine (trans-4f). ${ }^{1} \mathrm{H}$ NMR $[500 \mathrm{MHz}, \delta$ (ppm), $\mathrm{CDCl}_{3}$ ]: 7.38-7.35 (m, $\left.2 \mathrm{H}\right), 7.22-7.18(\mathrm{~m}, 2 \mathrm{H}), 7.01-6.98(\mathrm{~m}, 2$ $\mathrm{H}), 6.97-6.93(\mathrm{~m}, 1 \mathrm{H}), 6.92-6.89(\mathrm{~m}, 2 \mathrm{H}), 6.06(\mathrm{dd}, J=6.8,3.7 \mathrm{~Hz}$, $1 \mathrm{H}), 4.87-4.77(\mathrm{~m}, 1 \mathrm{H}), 3.81(\mathrm{~s}, 3 \mathrm{H}), 3.00-2.90(\mathrm{~m}, 1 \mathrm{H}), 2.84$ (ddd, $J=13.2,7.2,3.7 \mathrm{~Hz}, 1 \mathrm{H}) .{ }^{13} \mathrm{C}$ NMR $[126 \mathrm{MHz}, \delta(\mathrm{ppm})$, $\mathrm{CDCl}_{3}$ ]: 159.5, 151.0, 131.8, $130.0(\mathrm{q}, J=307.8 \mathrm{~Hz}), 128.8,127.9$, $122.4,115.4,114.5,82.3(\mathrm{q}, J=2.3 \mathrm{~Hz}), 67.7,55.5,46.3$. FTIR $[\bar{\nu}$ $\left.\left(\mathrm{cm}^{-1}\right)\right]: 3002,1512,1489,1248,1109,1030,794,693$. HRMS (EI) $\mathrm{m} /$ $z:\left[\mathrm{M}^{+\bullet}\right]$ calcd for $\mathrm{C}_{17} \mathrm{H}_{16} \mathrm{~F}_{3} \mathrm{NO}_{2} \mathrm{~S}$ 355.0854, found 355.0855. R: 0.10 (heptane $/ \mathrm{CH}_{2} \mathrm{Cl}_{2}, 4: 1$ ).

3-(3-Fluorophenyl)-2-phenyl-5-[(trifluoromethyl)sulfanyl]isoxazolidine (4g). According to the general procedure, the reaction of nitrone $6 \mathrm{~g}(52 \mathrm{mg}, 0.242 \mathrm{mmol})$ and alkene 2 in a $1.5 \mathrm{~mL}$ PTFE highpressure tube afforded isoxazolidine cis- $4 \mathrm{~g}$ ( $46.6 \mathrm{mg}, 0.136 \mathrm{mmol}, 56 \%)$ as a white solid, a mixture of cis- and trans- $4 \mathrm{~g}(27.8 \mathrm{mg}, 0.081,34 \%)$ as a yellow oil, and isoxazolidine trans-4g mixed with cis-4g and regioisomers $7 \mathrm{~g}(8.5 \mathrm{mg}, 0.025 \mathrm{mmol}, 10 \%)$ as a yellow solid, after column chromatography (heptane $/ \mathrm{CH}_{2} \mathrm{Cl}_{2}, 9: 1$ ). Total yield: $99 \%$.

rac-(3R,5R)-3-(3-Fluorophenyl)-2-phenyl-5-[(trifluoromethyl)sulfanyl]isoxazolidine (cis-4g). ${ }^{1} \mathrm{H}$ NMR $[500 \mathrm{MHz}, \delta$ (ppm), $\mathrm{CDCl}_{3}$ ]: $7.34(\mathrm{td}, J=8.1,5.9 \mathrm{~Hz}, 1 \mathrm{H}), 7.28-7.18(\mathrm{~m}, 4 \mathrm{H}), 7.06-$ $6.98(\mathrm{~m}, 2 \mathrm{H}), 6.98-6.93(\mathrm{~m}, 2 \mathrm{H}), 5.98(\mathrm{dd}, J=8.1,4.3 \mathrm{~Hz}, 1 \mathrm{H}), 4.41$ (dd, $J=9.1,6.8 \mathrm{~Hz}, 1 \mathrm{H}), 3.39$ (dddq, $J=13.7,9.1,8.1,0.9 \mathrm{~Hz}, 1 \mathrm{H}$ ), 2.33 (ddd, $J=13.7,6.8,4.3 \mathrm{~Hz}, 1 \mathrm{H}) .{ }^{13} \mathrm{C}$ NMR [126 MHz, $\delta(\mathrm{ppm})$, $\mathrm{CDCl}_{3}$ ]: $163.4(\mathrm{~d}, J=247.0 \mathrm{~Hz}), 148.6,142.5(\mathrm{~d}, J=7.2 \mathrm{~Hz}), 130.9$ (d, $J=8.3 \mathrm{~Hz}), 130.1(\mathrm{q}, J=307.9 \mathrm{~Hz}), 128.9,123.9,122.7(\mathrm{~d}, J=2.9 \mathrm{~Hz})$, $117.4,115.3(\mathrm{~d}, J=21.3 \mathrm{~Hz}), 114.1(\mathrm{~d}, J=22.6 \mathrm{~Hz}), 80.4(\mathrm{q}, J=2.4$ $\mathrm{Hz}), 67.5(\mathrm{~d}, J=1.9 \mathrm{~Hz}), 46.9 .{ }^{19} \mathrm{~F} \mathrm{NMR}\left[471 \mathrm{MHz}, \delta(\mathrm{ppm}), \mathrm{CDCl}_{3}\right]$ : -39.9. FTIR $\left[\bar{\nu}\left(\mathrm{cm}^{-1}\right)\right]: 3062,1594,1489,1448,1258,1111,1044$, 915, 833, 752, 690. HRMS (EI) $m / z$ : $\left[\mathrm{M}^{+\bullet}\right]$ calcd for $\mathrm{C}_{16} \mathrm{H}_{13} \mathrm{~F}_{4} \mathrm{NOS}$ 343.0654, found 343.0675. $R_{f}: 0.22$ (heptane $/ \mathrm{CH}_{2} \mathrm{Cl}_{2}, 17: 3$ ).

rac-(3R,5S)-3-(3-Fluorophenyl)-2-phenyl-5-[(trifluoromethyl)sulfanyl]isoxazolidine (trans-4g). ${ }^{1} \mathrm{H}$ NMR $[500 \mathrm{MHz}, \delta$ (ppm), $\mathrm{CDCl}_{3}$ ]: $7.35(\mathrm{td}, J=8.1,5.9 \mathrm{~Hz}, 1 \mathrm{H}), 7.25-7.20(\mathrm{~m}, 4 \mathrm{H}), 7.04-6.94$ $(\mathrm{m}, 4 \mathrm{H}), 6.05(\mathrm{dd}, J=6.8,4.0 \mathrm{~Hz}, 1 \mathrm{H}), 4.90(\mathrm{t}, J=7.4 \mathrm{~Hz}, 1 \mathrm{H}), 2.99-$ $2.91(\mathrm{~m}, 1 \mathrm{H}), 2.33$ (ddd, $J=13.2,7.4,4.0 \mathrm{~Hz}, 1 \mathrm{H}) .{ }^{13} \mathrm{C}$ NMR [126 $\left.\mathrm{MHz}, \delta(\mathrm{ppm}), \mathrm{CDCl}_{3}\right]: 163.4(\mathrm{~d}, J=247.1 \mathrm{~Hz}), 150.6,142.6(\mathrm{~d}, J=$
$7.2 \mathrm{~Hz}), 130.7(\mathrm{~d}, J=8.3 \mathrm{~Hz}), 128.9,122.6,122.2(\mathrm{~d}, J=2.8 \mathrm{~Hz}), 115.2$ $(\mathrm{d}, J=21.6 \mathrm{~Hz}), 115.1,113.7(\mathrm{~d}, J=22.7 \mathrm{~Hz}), 80.2(\mathrm{q}, J=2.5 \mathrm{~Hz}), 67.4$ $(\mathrm{d}, J=2.1 \mathrm{~Hz}), 46.0 .{ }^{19} \mathrm{~F}$ NMR $\left[471 \mathrm{MHz}, \delta(\mathrm{ppm}), \mathrm{CDCl}_{3}\right]:-39.4$. FTIR $\left[\bar{\nu}\left(\mathrm{cm}^{-1}\right)\right]: 3070,1593,1488,1451,1256,1109,947,787,691$. HRMS (EI) $m / z:\left[\mathrm{M}^{+\bullet}\right]$ calcd for $\mathrm{C}_{16} \mathrm{H}_{13} \mathrm{~F}_{4} \mathrm{NOS} 343.0654$, found 343.0667. $R_{f} 0.15$ (heptane $/ \mathrm{CH}_{2} \mathrm{Cl}_{2}, 17: 3$ ).

2-Phenyl-3-(3-tolyl)-5-[(trifluoromethyl)sulfanyl]isoxazolidine (4h). According to the general procedure, the reaction of nitrone $6 \mathrm{~h}$ (49 $\mathrm{mg}, 0.234 \mathrm{mmol}$ ) and alkene 2 in a $1.5 \mathrm{~mL}$ PTFE high-pressure tube afforded isoxazolidine cis- $4 \mathrm{~h}(46 \mathrm{mg}, 0.136 \mathrm{mmol}, 57 \%)$ as an off white solid and isoxazolidine trans- $4 \mathrm{~h}$ and regioisomers $7 \mathrm{~h}(26 \mathrm{mg}$, $0.077 \mathrm{mmol}, 31 \%)$ as a yellow solid, after column chromatography (heptane $\left./ \mathrm{CH}_{2} \mathrm{Cl}_{2}, 17: 3 \rightarrow 7: 3\right)$. Total yield: $88 \%$.

rac-(3R,5R)-2-Phenyl-3-(3-tolyl)-5-[(trifluoromethyl)sulfanyl]isoxazolidine (cis-4h). ${ }^{1} \mathrm{H}$ NMR [500 MHz, $\delta$ (ppm), $\left.\mathrm{CDCl}_{3}\right]$ : 7.31$7.28(\mathrm{~m}, 1 \mathrm{H}), 7.27-7.24(\mathrm{~m}, 2 \mathrm{H}), 7.22-7.17(\mathrm{~m}, 2 \mathrm{H}), 7.14-7.10(\mathrm{~m}$, $1 \mathrm{H}), 7.02-6.94(\mathrm{~m}, 3 \mathrm{H}), 5.98(\mathrm{dd}, J=8.1,4.4 \mathrm{~Hz}, 1 \mathrm{H}), 4.35(\mathrm{dd}, J=$ 9.0, $7.2 \mathrm{~Hz}, 1 \mathrm{H}$ ), 3.37 (dddq, $J=13.6,9.0,8.1,0.9 \mathrm{~Hz}, 1 \mathrm{H}$ ), $2.38-2.32$ $(\mathrm{m}, 1 \mathrm{H}), 2.36(\mathrm{~s}, 3 \mathrm{H}) .{ }^{13} \mathrm{C}$ NMR $\left[126 \mathrm{MHz}, \delta(\mathrm{ppm}), \mathrm{CDCl}_{3}\right]: 148.9$, $139.7,139.1,130.2(\mathrm{q}, J=307.7 \mathrm{~Hz}), 129.1,129.1,128.8,127.6,124.2$, $123.6,117.5,80.5(\mathrm{q}, J=2.2 \mathrm{~Hz}), 68.3,47.2,21.6$. FTIR $\left[\bar{\nu}\left(\mathrm{cm}^{-1}\right)\right]$ : $3028,1599,1489,1040,1001,839,737,724,692$. HRMS (EI) $\mathrm{m} / z$ : $\left[\mathrm{M}^{+\bullet}\right]$ calcd for $\mathrm{C}_{17} \mathrm{H}_{16} \mathrm{~F}_{3} \mathrm{NOS} 339.0905$, found 339.0925. $R_{f}: 0.41$ (heptane $/ \mathrm{CH}_{2} \mathrm{Cl}_{2}, 4: 1$ ).

rac-(3R,5S)-2-Phenyl-3-(3-tolyl)-5-[(trifluoromethyl)sulfanyl]isoxazolidine (trans-4h). ${ }^{1} \mathrm{H}$ NMR $\left[500 \mathrm{MHz}, \delta(\mathrm{ppm}), \mathrm{CDCl}_{3}\right]$ : 7.31-7.18 (m, 5 H), 7.14-7.10 (m, 1 H), 7.03-6.98 (m, $2 \mathrm{H}), 6.98-$ $6.91(\mathrm{~m}, 1 \mathrm{H}), 6.06(\mathrm{dd}, J=6.8,3.7 \mathrm{~Hz}, 1 \mathrm{H}), 4.85(\mathrm{t}, J=7.3 \mathrm{~Hz}, 1 \mathrm{H})$, 2.95 (dddq, $J=13.2,7.6,6.8,0.9 \mathrm{~Hz}, 1 \mathrm{H}$ ), 2.86 (ddd, $J=13.2,7.3$, 3.7 $\mathrm{Hz}, 1 \mathrm{H}), 2.36(\mathrm{~s}, 3 \mathrm{H}) .{ }^{13} \mathrm{C}$ NMR [126 MHz, $\left.\delta(\mathrm{ppm}), \mathrm{CDCl}_{3}\right]: 151.2$, $139.9,139.0,130.0$ (q, $J=307.8 \mathrm{~Hz}$ ), 129.0, 128.9, 128.8, 127.1, 123.7, $122.3,115.1,82.4(\mathrm{q}, J=2.1 \mathrm{~Hz}), 68.1,46.4,21.6 .{ }^{19} \mathrm{~F}$ NMR $[471 \mathrm{MHz}$, $\left.\delta(\mathrm{ppm}), \mathrm{CDCl}_{3}\right]:-39.3$. FTIR $\left[\bar{\nu}\left(\mathrm{cm}^{-1}\right)\right]: 3029,1598,1489,1150$, $1111,1045,785,692$. HRMS (EI) $\mathrm{m} / z$ : $\left[\mathrm{M}^{+\bullet}\right]$ calcd for $\mathrm{C}_{17} \mathrm{H}_{16} \mathrm{~F}_{3} \mathrm{NOS}$ 339.0905, found 339.0931. $R_{f} 0.34$ (heptane $/ \mathrm{CH}_{2} \mathrm{Cl}_{2}, 4: 1$ ).

Methyl 3-\{2-Phenyl-5-[(trifluoromethyl)sulfanyl]isoxazolidin-3yl\}benzoate (4i). According to the general procedure, the reaction of nitrone $6 \mathbf{i}(26 \mathrm{mg}, 0.102 \mathrm{mmol})$ and alkene 2 in a $1 \mathrm{~mL}$ PTFE highpressure tube, containing approximately $150 \mu \mathrm{L}$ of glass beads, afforded isoxazolidine cis-4i $(17.3 \mathrm{mg}, 0.045 \mathrm{mmol}, 44 \%)$ as an off-white solid and a mixture of isoxazolidines cis- and trans- $4 \mathbf{i}$ and regioisomers $7 \mathbf{i}$ (18.2 $\mathrm{mg}, 0.047 \mathrm{mmol}, 47 \%$ ) as a brown-yellow solid, after column chromatography (heptane $/ \mathrm{CH}_{2} \mathrm{Cl}_{2}, 3: 2$ ). Total yield: $91 \%$.

Methyl 3-\{rac-(3R,5R)-2-Phenyl-5-[(trifluoromethyl)sulfanyl]isoxazolidin-3-yl\}benzoate (cis-4i). ${ }^{1} \mathrm{H}$ NMR $[500 \mathrm{MHz}, \delta(\mathrm{ppm})$, $\left.\mathrm{CDCl}_{3}\right]: 8.11(\mathrm{t}, J=1.8 \mathrm{~Hz}, 1 \mathrm{H}), 8.01(\mathrm{dt}, J=7.7,1.4 \mathrm{~Hz}, 1 \mathrm{H}), 7.76$ (dddd, $J=7.7,1.8,1.2,0.5 \mathrm{~Hz}, 1 \mathrm{H}), 7.48(\mathrm{t}, J=7.7 \mathrm{~Hz}, 1 \mathrm{H}), 7.24-$ $7.19(\mathrm{~m}, 2 \mathrm{H}), 7.07-7.00(\mathrm{~m}, 1 \mathrm{H}), 6.98-6.94(\mathrm{~m}, 2 \mathrm{H}), 6.01(\mathrm{dd}, J=$ 8.0, $4.5 \mathrm{~Hz}, 1 \mathrm{H}), 4.50$ (dd, $J=8.9,7.0 \mathrm{~Hz}, 1 \mathrm{H}), 3.93(\mathrm{~s}, 3 \mathrm{H}), 3.43$ (dddq, $J=13.6,8.9,8.0,0.9 \mathrm{~Hz}, 1 \mathrm{H}), 2.42-2.30(\mathrm{~m}, 1 \mathrm{H}) .{ }^{13} \mathrm{C}$ NMR [126 MHz, $\left.\delta(\mathrm{ppm}), \mathrm{CDCl}_{3}\right]: 166.8,148.6,140.4,131.6,131.1,130.0$ $(\mathrm{q}, J=307.7 \mathrm{~Hz}), 129.6,129.0,128.3,124.0,117.6,80.4(\mathrm{q}, J=2.5 \mathrm{~Hz})$, 67.9, 52.4, 46.9. ${ }^{19} \mathrm{~F}$ NMR $\left[471 \mathrm{MHz}, \delta(\mathrm{ppm}), \mathrm{CDCl}_{3}\right]:-39.9$. FTIR $\left[\bar{\nu}\left(\mathrm{cm}^{-1}\right)\right]: 3015,1729,1598,1489,1301,1247,1114,1025,786,692$, 674. HRMS (EI) $m / z$ : $\left[\mathrm{M}^{+\bullet}\right]$ calcd for $\mathrm{C}_{18} \mathrm{H}_{16} \mathrm{~F}_{3} \mathrm{NO}_{3} \mathrm{~S} 383.0803$, found 383.0824. $R_{f} 0.27$ (heptane/ $\mathrm{CH}_{2} \mathrm{Cl}_{2}, 3: 2$ ).

Methyl-3-\{rac-(3R,5S)-2-phenyl-5-[(trifluoromethyl)sulfanyl]isoxazolidin-3-yl\}benzoate (trans-4i). ${ }^{1} \mathrm{H}$ NMR $[500 \mathrm{MHz}, \delta(\mathrm{ppm})$, $\left.\mathrm{CDCl}_{3}\right]: 8.12(\mathrm{t}, J=1.8 \mathrm{~Hz}, 1 \mathrm{H}), 8.02-7.98(\mathrm{~m}, 1 \mathrm{H}), 7.69(\mathrm{dt}, J=7.8$, $1.6 \mathrm{~Hz}, 1 \mathrm{H}), 7.47(\mathrm{t}, J=7.7,1 \mathrm{H}), 7.26-7.18(\mathrm{~m}, 2 \mathrm{H}), 7.00-6.98(\mathrm{~m}$, $2 \mathrm{H}), 6.97-6.94(\mathrm{~m}, 1 \mathrm{H}), 6.06(\mathrm{dd}, J=6.7,4.0 \mathrm{~Hz}, 1 \mathrm{H}), 4.96(\mathrm{t}, J=$ $7.3 \mathrm{~Hz}, 1 \mathrm{H}), 3.93(\mathrm{~s}, 3 \mathrm{H}), 3.01-2.94(\mathrm{~m}, 1 \mathrm{H}), 2.91$ (ddd, $J=13.3$, 7.4, $4.0 \mathrm{~Hz}, 1 \mathrm{H}) .{ }^{13} \mathrm{C}$ NMR $\left[126 \mathrm{MHz}, \delta(\mathrm{ppm}), \mathrm{CDCl}_{3}\right]: 166.8,150.7$, $140.5,131.19,131.14,130.0(\mathrm{q}, J=307.9 \mathrm{~Hz}), 129.57,129.55,128.9$, $127.8,122.6,115.2,82.3(\mathrm{q}, J=2.2 \mathrm{~Hz}), 67.6,52.4,46.1 .{ }^{19} \mathrm{~F}$ NMR $[471$ $\left.\mathrm{MHz}, \delta(\mathrm{ppm}), \mathrm{CDCl}_{3}\right]:-39.4$. FTIR $\left[\bar{\nu}\left(\mathrm{cm}^{-1}\right)\right]: 3064,1720,1598$, $1489,1285,1107,1020,799,751,692$. HRMS (EI) $\mathrm{m} / z:\left[\mathrm{M}^{+\bullet}\right]$ calcd for $\mathrm{C}_{18} \mathrm{H}_{16} \mathrm{~F}_{3} \mathrm{NO}_{3} \mathrm{~S}$ 383.0803, found 383.0810. $R_{f}: 0.22$ (heptane/ $\mathrm{CH}_{2} \mathrm{Cl}_{2}, 3: 2$ ). 
3-(3-Methoxyphenyl)-2-phenyl-5-[(trifluoromethyl)sulfanyl]isoxazolidine (4j). According to the general procedure, the reaction of nitrone $6 \mathbf{j}(50 \mathrm{mg}, 0.190 \mathrm{mmol})$ and alkene 2 in a $1.5 \mathrm{~mL}$ PTFE highpressure tube, containing approximately $300 \mu \mathrm{L}$ of glass beads, afforded isoxazolidine cis-4j $(33.8 \mathrm{mg}, 0.095 \mathrm{mmol}, 53 \%)$ as a white solid, a mixture of isoxazolidines cis- and trans- $4 \mathbf{j}$ ( $3 \mathrm{mg}, 0.0084 \mathrm{mmol}, 5 \%)$ as a yellow oil and isoxazolidines trans- $4 \mathbf{j}$ and $7 \mathbf{j}(18.2 \mathrm{mg}, 0.051 \mathrm{mmol}$, $22 \%$ ) as a yellow oil, after column chromatography (heptane $/ \mathrm{CH}_{2} \mathrm{Cl}_{2}$, 7:3). Total yield: $80 \%$.

rac-(3R,5R)-3-(3-Methoxyphenyl)-2-phenyl-5-[(trifluoromethyl)sulfanyl]isoxazolidine (cis-4j). ${ }^{1} \mathrm{H} \mathrm{NMR}\left[400 \mathrm{MHz}, \delta(\mathrm{ppm}), \mathrm{CDCl}_{3}\right]$ : $7.29(\mathrm{t}, J=7.9 \mathrm{~Hz}, 1 \mathrm{H}), 7.24-7.17(\mathrm{~m}, 2 \mathrm{H}), 7.09-6.95(\mathrm{~m}, 5 \mathrm{H}), 6.85$ (ddd, $J=8.3,2.7,1.1 \mathrm{~Hz}, 1 \mathrm{H}), 5.99(\mathrm{dd}, J=8.1,4.3 \mathrm{~Hz}, 1 \mathrm{H}), 4.37$ (dd, $J=9.1,7.0 \mathrm{~Hz}, 1 \mathrm{H}), 3.81(\mathrm{~s}, 3 \mathrm{H}), 3.43-3.32(\mathrm{~m}, 1 \mathrm{H}), 2.36(\mathrm{ddd}, J=$ 13.7, 7.0, $4.3 \mathrm{~Hz}, 1 \mathrm{H}) .{ }^{13} \mathrm{C}$ NMR [101 MHz, $\delta(\mathrm{ppm}), \mathrm{CDCl}_{3}$ ]: 160.4, $148.9,141.6,130.3,130.2(q, J=307.7 \mathrm{~Hz}), 128.8,123.6,119.3,117.3$, $113.8,112.4,80.5(\mathrm{q}, J=2.5 \mathrm{~Hz}), 68.0,55.4,47.2(\mathrm{q}, J=1.2 \mathrm{~Hz}) .{ }^{19} \mathrm{~F}$ NMR $\left[377 \mathrm{MHz}, \delta(\mathrm{ppm}), \mathrm{CDCl}_{3}\right]:-39.9$. FTIR $\left[\bar{\nu}\left(\mathrm{cm}^{-1}\right)\right]: 3006$, $2838,1599,1490,1117,757,694$. HRMS (EI) $m / z:\left[\mathrm{M}^{+\bullet}\right]$ calcd for $\mathrm{C}_{17} \mathrm{H}_{16} \mathrm{~F}_{3} \mathrm{NO}_{2} \mathrm{~S}$ 355.0854, found 355.0868. $R_{f}: 0.16$ (heptane $/ \mathrm{CH}_{2} \mathrm{Cl}_{2}$, $7: 3)$.

rac-(3R,5S)-3-(3-Methoxyphenyl)-2-phenyl-5-[(trifluoromethyl)sulfanyl]isoxazolidine (trans-4j). ${ }^{1} \mathrm{H}$ NMR $[400 \mathrm{MHz}, \delta$ (ppm), $\mathrm{CDCl}_{3}$ ]: $7.29(\mathrm{t}, J=8.1 \mathrm{~Hz}, 1 \mathrm{H}), 7.25-7.19(\mathrm{~m}, 2 \mathrm{H}), 7.07-6.92(\mathrm{~m}, 5$ $\mathrm{H}), 6.89-6.81(\mathrm{~m}, 1 \mathrm{H}), 6.06(\mathrm{dd}, J=6.8,3.8,1 \mathrm{H}), 4.86(\mathrm{t}, J=7.4 \mathrm{~Hz}$, $1 \mathrm{H}), 3.81(\mathrm{~s}, 3 \mathrm{H}), 2.96$ (dddq, $J=13.3,7.6,6.8,0.9 \mathrm{~Hz}, 1 \mathrm{H}), 2.87$ (ddd, $J=13.3,7.3,3.8 \mathrm{~Hz}, 1 \mathrm{H}) .{ }^{13} \mathrm{C}$ NMR $[101 \mathrm{MHz}, \delta(\mathrm{ppm})$, $\left.\mathrm{CDCl}_{3}\right]: 160.3,151.0,141.6,130.2,130.0(\mathrm{q}, J=307.8 \mathrm{~Hz}), 128.8$, 122.3, 118.8, 115.1, 113.7, 112.0, $82.3(\mathrm{q}, J=2.3 \mathrm{~Hz}), 68.0,55.5,46.3$. ${ }^{19} \mathrm{~F}$ NMR $\left[377 \mathrm{MHz}, \delta(\mathrm{ppm}), \mathrm{CDCl}_{3}\right]:-39.3$. FTIR $\left[\bar{\nu}\left(\mathrm{cm}^{-1}\right)\right]$ : 3064, 1720, 1598, 1489, 1347, 1107, 1020, 799, 750, 692. HRMS (ESITOF) $m / z:[\mathrm{M}+\mathrm{H}]^{+}$calcd for $\mathrm{C}_{17} \mathrm{H}_{17} \mathrm{~F}_{3} \mathrm{NO}_{2} \mathrm{~S}$ 356.0932, found 356.0939. $R_{f}: 0.13$ (heptane $/ \mathrm{CH}_{2} \mathrm{Cl}_{2}, 7: 3$ ).

2-Phenyl-3-[3-(trifluoromethyl)phenyl]-5-[(trifluoromethyl)sulfanyl]isoxazolidine (4k). According to the general procedure, the reaction of nitrone $6 \mathrm{k}(49 \mathrm{mg}, 0.234 \mathrm{mmol})$ and alkene 2 in a $1.5 \mathrm{~mL}$ PTFE high-pressure tube afforded a mixture of isoxazolidines cis- and trans- $4 \mathrm{k}$ and $7 \mathrm{k}(87.4 \mathrm{mg}, 0.222 \mathrm{mmol}, 95 \%)$ as a white-yellow solid, after column chromatography (heptane/ $\mathrm{CH}_{2} \mathrm{Cl}_{2}, 17: 3 \rightarrow 7: 3$ ). FTIR $[\bar{\nu}$ $\left.\left(\mathrm{cm}^{-1}\right)\right]: 2927,1598,1490,1263,1144,1045$. HRMS (EI) $\mathrm{m} / z:\left[\mathrm{M}^{+\bullet}\right]$ calcd for $\mathrm{C}_{17} \mathrm{H}_{13} \mathrm{~F}_{6} \mathrm{NOS} 393.0622$, found 393.0639. $R_{f}$ 0.38 (heptane/ $\left.\mathrm{CH}_{2} \mathrm{Cl}_{2}, 4: 1\right)$. Total yield: $95 \%$.

rac-(3R,5R)-2-Phenyl-3-[3-(trifluoromethyl)phenyl]-5[(trifluoromethyl)sulfanyl]isoxazolidine (cis-4k). ${ }^{1} \mathrm{H}$ NMR $[500 \mathrm{MHz}$, $\delta$ (ppm), $\mathrm{CDCl}_{3}$ ]: 7.73-7.69 (m, $\left.2 \mathrm{H}\right), 7.60-7.56(\mathrm{~m}, 1 \mathrm{H}), 7.54-7.48$ (m, $1 \mathrm{H}), 7.26-7.20(\mathrm{~m}, 2 \mathrm{H}), 7.05-7.00(\mathrm{~m}, 1 \mathrm{H}), 6.98-6.93(\mathrm{~m}, 2$ H), 5.99 (dd, $J=8.0,4.5 \mathrm{~Hz}, 1 \mathrm{H}), 4.51(\mathrm{dd}, J=9.0,6.7 \mathrm{~Hz}, 1 \mathrm{H}), 3.41$ (dddq, $J=13.6,9.0,8.0,0.8 \mathrm{~Hz}, 1 \mathrm{H}), 2.37-2.28(\mathrm{~m}, 1 \mathrm{H}) .{ }^{13} \mathrm{C}$ NMR [126 MHz, $\left.\delta(\mathrm{ppm}), \mathrm{CDCl}_{3}\right]: 148.6,141.1,131.6(\mathrm{q}, J=32.4 \mathrm{~Hz})$, $130.4(\mathrm{q}, J=1.2 \mathrm{~Hz}), 129.9(\mathrm{q}, J=308.0 \mathrm{~Hz}), 129.9,129.0,125.2(\mathrm{q}, J=$ $3.8 \mathrm{~Hz}), 124.0(\mathrm{q}, J=272.4 \mathrm{~Hz}), 124.1,123.9(\mathrm{q}, J=3.8 \mathrm{~Hz}), 117.4$, $80.4(\mathrm{q}, J=2.5 \mathrm{~Hz}), 67.8,46.8$.

rac-(3R,5S)-2-Phenyl-3-[3-(trifluoromethyl)phenyl]-5-[(trifluoromethyl)sulfanyl]isoxazolidine (trans-4k). ${ }^{1} \mathrm{H}$ NMR $[500 \mathrm{MHz}, \delta$ (ppm), $\mathrm{CDCl}_{3}$ ]: 7.73-7.70 (m, $\left.1 \mathrm{H}\right), 7.68-7.64(\mathrm{~m}, 1 \mathrm{H}), 7.60-7.56$ (m, $1 \mathrm{H}), 7.53-7.48(\mathrm{~m}, 1 \mathrm{H}), 7.26-7.20(\mathrm{~m}, 2 \mathrm{H}), 6.92-6.88(\mathrm{~m}, 3$ $\mathrm{H}), 6.06-6.02(\mathrm{~m}, 1 \mathrm{H}), 4.96(\mathrm{t}, J=7.2 \mathrm{~Hz}, 1 \mathrm{H}), 2.98-2.86(\mathrm{~m}, 2 \mathrm{H})$. ${ }^{13} \mathrm{C}$ NMR [126 MHz, $\left.\delta(\mathrm{ppm}), \mathrm{CDCl}_{3}\right]: 150.6,141.1,131.6(\mathrm{q}, J=32.4$ $\mathrm{Hz}), 130.4(\mathrm{q}, J=1.2 \mathrm{~Hz}), 129.7,128.9,125.1(\mathrm{q}, J=4.0 \mathrm{~Hz}), 123.5(\mathrm{q}$, $J=3.8 \mathrm{~Hz}), 122.7,115.2,82.3(\mathrm{q}, J=2.4 \mathrm{~Hz}), 67.5,46.0$.

2-Phenyl-3-(2-tolyl)-5-[(trifluoromethyl)sulfanyl]isoxazolidine (4I). According to the general procedure, the reaction of nitrone 61 (49 $\mathrm{mg}, 0.234 \mathrm{mmol}$ ) and alkene $2 \mathrm{in}$ a $1.5 \mathrm{~mL}$ PTFE high-pressure tube afforded isoxazolidine cis-4l (1.9 mg, $0.0056 \mathrm{mmol}$; used for characterization) as a colorless oil and a mixture of isoxazolidines cisand trans-41 (79.5 mg, $0.234 \mathrm{mmol}, 99 \%)$ as a yellow solid, after column chromatography (heptane $\left./ \mathrm{CH}_{2} \mathrm{Cl}_{2}, 4: 1\right)$. FTIR $\left[\bar{\nu}\left(\mathrm{cm}^{-1}\right)\right]: 3025$, $1596,1489,1258,1109,1041,972,754,695$. HRMS (EI) $\mathrm{m} / z:\left[\mathrm{M}^{+\bullet}\right]$ calcd for $\mathrm{C}_{17} \mathrm{H}_{16} \mathrm{~F}_{3} \mathrm{NOS} 339.0905$, found 339.0923. Rf: 0.23 (heptane/ $\mathrm{CH}_{2} \mathrm{Cl}_{2}, 4: 1$ ). Total yield: $99 \%$.
rac-(3R,5R)-2-Phenyl-3-(2-tolyl)-5-[(trifluoromethyl)sulfanyl]isoxazolidine (cis-4l). ${ }^{1} \mathrm{H}$ NMR [500 $\mathrm{MHz}, \delta(\mathrm{ppm}), \mathrm{CDCl}_{3}$ ]: 7.72 $(\mathrm{dd}, J=7.2,1.7 \mathrm{~Hz}, 1 \mathrm{H}), 7.26-7.17(\mathrm{~m}, 5 \mathrm{H}), 7.00(\mathrm{tt}, J=7.4,1.2 \mathrm{~Hz}$, $1 \mathrm{H}), 6.96-6.88(\mathrm{~m}, 2 \mathrm{H}), 6.01(\mathrm{dd}, J=8.1,4.2 \mathrm{~Hz}, 1 \mathrm{H}), 4.61(\mathrm{dd}, J=$ 9.1, 7.1 Hz, $1 \mathrm{H}$ ), 3.44 (dddq, $J=13.5,9.1,8.1,0.9 \mathrm{~Hz}, 1 \mathrm{H}), 2.35$ (s, 3 H), 2.25 (ddd, $J=13.5,7.1,4.2 \mathrm{~Hz}, 1 \mathrm{H}) .{ }^{13} \mathrm{C}$ NMR [126 MHz, $\delta$ $\left.(\mathrm{ppm}), \mathrm{CDCl}_{3}\right]: 148.9,138.0,134.6,130.8,130.1(\mathrm{q}, J=307.4 \mathrm{~Hz})$, $128.9,127.8,127.3,126.6,123.5,117.2,80.5$ (q, $J=2.2 \mathrm{~Hz}), 64.5,45.6$, 19.7. ${ }^{19} \mathrm{~F}$ NMR $\left[471 \mathrm{MHz}, \delta(\mathrm{ppm}), \mathrm{CDCl}_{3}\right]:-40.0$.

rac-(3R,5S)-2-Phenyl-3-(2-tolyl)-5-[(trifluoromethyl)sulfanyl]isoxazolidine (trans-4l). ${ }^{1} \mathrm{H}$ NMR $\left[500 \mathrm{MHz}, \delta(\mathrm{ppm}), \mathrm{CDCl}_{3}\right]$ : 7.65-7.62 (m, 1 H), 7.27-7.15 (m, $5 \mathrm{H}), 6.97-6.93(\mathrm{~m}, 3 \mathrm{H}), 6.06$ (dd, $J=6.7,3.9 \mathrm{~Hz}, 1 \mathrm{H}), 5.04(\mathrm{t}, J=7.5 \mathrm{~Hz}, 1 \mathrm{H}), 2.89$ (ddd, $J=13.1$, 7.5, $3.9 \mathrm{~Hz}, 1 \mathrm{H}), 2.80$ (dddq, $J=13.1,7.5,6.7,0.9 \mathrm{~Hz}, 1 \mathrm{H}), 2.39(\mathrm{~s}, 3$ H). ${ }^{13} \mathrm{C}$ NMR $\left[126 \mathrm{MHz}, \delta(\mathrm{ppm}), \mathrm{CDCl}_{3}\right]$ : $151.1,137.8,134.3,130.8$, $128.7,127.8,127.0,126.2,122.1,114.7,82.2$ (q, $J=2.3 \mathrm{~Hz}$ ), 65.3, 44.6, 19.4 .

3-(2-Methoxyphenyl)-2-phenyl-5-[(trifluoromethyl)sulfanyl]isoxazolidine $(4 \mathrm{~m})$. According to the general procedure, the reaction of nitrone $6 \mathrm{~m}(53 \mathrm{mg}, 0.234 \mathrm{mmol})$ and alkene 2 in a $1.5 \mathrm{~mL}$ PTFE highpressure tube afforded isoxazolidine cis- $4 \mathrm{~m}(35.6 \mathrm{mg}, 0.100 \mathrm{mmol}$, $43 \%)$ as an off white solid and a mixture of isoxazolidines cis- and trans4m (29.6 mg, $0.083 \mathrm{mmol}, 28 \%)$ as a yellow solid, after column chromatography (heptane $/ \mathrm{CH}_{2} \mathrm{Cl}_{2}, 7: 3$ ). Total yield: $71 \%$.

rac-(3R,5R)-3-(2-Methoxyphenyl)-2-phenyl-5-[(trifluoromethyl)sulfanyl]isoxazolidine (cis-4m). ${ }^{1} \mathrm{H}$ NMR $[500 \mathrm{MHz}, \delta$ (ppm), $\mathrm{CDCl}_{3}$ ]: 7.65 (dd, $J=7.6,1.7 \mathrm{~Hz}, 1 \mathrm{H}$ ), 7.28 (ddd, $J=8.2,7.5,1.8 \mathrm{~Hz}, 1$ H), 7.25-7.19 (m, 2 H), 7.01-6.94 (m, $4 \mathrm{H}), 6.91(\mathrm{dd}, J=8.3,1.0 \mathrm{~Hz}$, $1 \mathrm{H}), 5.94(\mathrm{dd}, J=8.0,4.5 \mathrm{~Hz}, 1 \mathrm{H}), 4.87(\mathrm{dd}, J=9.1,6.1 \mathrm{~Hz}, 1 \mathrm{H}), 3.86$ (s, $3 \mathrm{H}$ ), 3.40 (dddq, $J=13.6,9.1,8.0,0.9 \mathrm{~Hz}, 1 \mathrm{H}), 2.30-2.20$ (m, 1 H). ${ }^{13} \mathrm{C}$ NMR $\left[126 \mathrm{MHz}, \delta(\mathrm{ppm}), \mathrm{CDCl}_{3}\right]: 156.3,149.4,130.3$ (q, $J=$ $307.7 \mathrm{~Hz}), 128.9,128.4,127.5,123.1,121.3,116.7,110.5,80.6$ (q, $J=$ $2.4 \mathrm{~Hz}), 62.1,55.5,45.0$. FTIR $\left[\bar{\nu}\left(\mathrm{cm}^{-1}\right)\right]: 3075,1599,1490,1243$, $1106,1027,897,751,664$. HRMS (EI) $\mathrm{m} / z:\left[\mathrm{M}^{+\bullet}\right]$ calcd for $\mathrm{C}_{17} \mathrm{H}_{16} \mathrm{~F}_{3} \mathrm{NO}_{2} \mathrm{~S} 355.0854$, found 355.0875. $R_{f}: 0.19$ (heptane $/ \mathrm{CH}_{2} \mathrm{Cl}_{2}$, $7: 3)$.

rac-(3R,5S)-3-(2-Methoxyphenyl)-2-phenyl-5-[(trifluoromethyl)sulfanyl]isoxazolidine (trans-4m). ${ }^{1} \mathrm{H}$ NMR $[500 \mathrm{MHz}, \delta(\mathrm{ppm})$, $\mathrm{CDCl}_{3}$ ] : 7.59 (dd, $\left.J=7.6,1.5 \mathrm{~Hz}, 1 \mathrm{H}\right), 7.31-7.26(\mathrm{~m}, 2 \mathrm{H}), 7.06-7.02$ $(\mathrm{m}, 2 \mathrm{H}), 7.00-6.88(\mathrm{~m}, 4 \mathrm{H}), 5.99-5.96(\mathrm{~m}, 1 \mathrm{H}), 5.26(\mathrm{dd}, J=7.5$, $5.8 \mathrm{~Hz}, 1 \mathrm{H}), 3.88(\mathrm{~s}, 3 \mathrm{H}), 2.90(\mathrm{ddd}, J=12.7,7.5,5.0 \mathrm{~Hz}, 1 \mathrm{H}), 2.84-$ $2.76(\mathrm{~m}, 1 \mathrm{H}) .{ }^{13} \mathrm{C}$ NMR $\left[126 \mathrm{MHz}, \delta(\mathrm{ppm}), \mathrm{CDCl}_{3}\right]: 156.3,151.2$, 128.9, 128.8, 128.1, 127.2, 122.0, 121.1, 114.9, 110.4, 82.4 (q, $J=2.2$ $\mathrm{Hz}), 62.9,55.5$, 43.7. The carbon signal of $\mathrm{SCF}_{3}$ was not observed. FTIR $\left[\bar{\nu}\left(\mathrm{cm}^{-1}\right)\right]: 2839,1599,1490,1256,1106,1026,751,692$. HRMS (EI) $\mathrm{m} / z$ : $\left[\mathrm{M}^{+\bullet}\right]$ calcd for $\mathrm{C}_{17} \mathrm{H}_{16} \mathrm{~F}_{3} \mathrm{NO}_{2} \mathrm{~S} 355.0854$, found 355.0862. $R_{f}: 0.15$ (heptane $/ \mathrm{CH}_{2} \mathrm{Cl}_{2}, 7: 3$ ).

2-Phenyl-3-(thiophen-3-yl)-5-[(trifluoromethyl)sulfanyl]isoxazolidine (4n). According to the general procedure, the reaction of nitrone $6 \mathrm{n}$ ( $48 \mathrm{mg}, 0.234 \mathrm{mmol})$ and alkene 2 in a $1.5 \mathrm{~mL}$ PTFE highpressure tube afforded a mixture of isoxazolidines cis- and trans-4n (68.4 $\mathrm{mg}, 0.206 \mathrm{mmol}, 88 \%$ ) as a yellow solid, after column chromatography (heptane $\left./ \mathrm{CH}_{2} \mathrm{Cl}_{2}, 3: 2\right)$. FTIR $\left[\bar{\nu}\left(\mathrm{cm}^{-1}\right)\right]: 3095$, $1598,1489,1257,1163,1110,1031,837,792,754,692$. HRMS (EI) $m / z:\left[\mathrm{M}^{+\bullet}\right]$ calcd for $\mathrm{C}_{14} \mathrm{H}_{12} \mathrm{~F}_{3} \mathrm{NOS}_{2} 331.0312$, found 331.0330. $R_{f}$. 0.19 (heptane $/ \mathrm{CH}_{2} \mathrm{Cl}_{2}, 17: 3$ ). Total yield: $88 \%$.

rac-(3R,5R)-2-Phenyl-3-(thiophen-3-yl)-5-[(trifluoromethyl)sulfanyl]isoxazolidine (cis-4n). ${ }^{1} \mathrm{H}$ NMR $[500 \mathrm{MHz}, \delta$ (ppm), $\left.\mathrm{CDCl}_{3}\right]: 7.36(\mathrm{dd}, J=5.0,3.0 \mathrm{~Hz}, 1 \mathrm{H}), 7.30-7.27(\mathrm{~m}, 1 \mathrm{H}), 7.25-$ $7.20(\mathrm{~m}, 2 \mathrm{H}), 7.19(\mathrm{dd}, J=5.0,1.3 \mathrm{~Hz}, 1 \mathrm{H}), 7.02(\mathrm{tt}, J=7.4,1.2 \mathrm{~Hz}, 1$ H), 7.00-6.96 (m, $2 \mathrm{H}), 6.00(\mathrm{dd}, J=8.2,4.2 \mathrm{~Hz}, 1 \mathrm{H}), 4.51(\mathrm{dd}, J=$ $8.8,6.7 \mathrm{~Hz}, 1 \mathrm{H}$ ), $3.36-3.28(\mathrm{~m}, 1 \mathrm{H}), 2.38$ (ddd, $J=13.6,6.7,4.2 \mathrm{~Hz}, 1$ H). ${ }^{13} \mathrm{C}$ NMR $\left[126 \mathrm{MHz}, \delta(\mathrm{ppm}), \mathrm{CDCl}_{3}\right]: 148.9,140.4,130.2(\mathrm{q}, J=$ $307.7 \mathrm{~Hz}), 128.8,127.3,126.1,123.9,122.7,117.5,80.5$ (q, $J=2.5 \mathrm{~Hz})$, 64.6, 45.9.

rac-(3R,5S)-2-Phenyl-3-(thiophen-3-yl)-5-[(trifluoromethyl)sulfanyl]isoxazolidine (trans-4n). ${ }^{1} \mathrm{H}$ NMR $[500 \mathrm{MHz}, \delta$ (ppm), $\left.\mathrm{CDCl}_{3}\right]$ : $7.32(\mathrm{dd}, J=5.0,3.0 \mathrm{~Hz}, 1 \mathrm{H}), 7.30-7.27(\mathrm{~m}, 1 \mathrm{H}), 7.26-7.20$ $(\mathrm{m}, 2 \mathrm{H}), 7.09(\mathrm{dd}, J=5.0,1.4 \mathrm{~Hz}, 1 \mathrm{H}), 7.06-6.95(\mathrm{~m}, 3 \mathrm{H}), 6.03(\mathrm{dd}$, $J=7.2,4.9 \mathrm{~Hz}, 1 \mathrm{H}), 5.01(\mathrm{t}, J=6.5 \mathrm{~Hz}, 1 \mathrm{H}), 2.96(\mathrm{dt}, J=13.0,6.4 \mathrm{~Hz}$, $1 \mathrm{H}), 2.78$ (ddd, $J=12.7,7.3,4.7 \mathrm{~Hz}, 1 \mathrm{H}) .{ }^{13} \mathrm{C}$ NMR $[126 \mathrm{MHz}, \delta$ 
(ppm), $\left.\mathrm{CDCl}_{3}\right]: 150.2,140.6,130.2(\mathrm{q}, J=307.7 \mathrm{~Hz}), 128.8,127.0$, $125.8,122.7,122.4,115.7,81.9(\mathrm{q}, J=2.2 \mathrm{~Hz}), 64.2,44.6$.

3-(1 H-Indol-2-yl)-2-phenyl-5-[(trifluoromethyl)sulfanyl]isoxazolidine (40). According to the general procedure, the reaction of nitrone 60 ( $56 \mathrm{mg}, 0.237 \mathrm{mmol})$ and alkene 2 in a $1.5 \mathrm{~mL}$ PTFE highpressure tube afforded isoxazolidines cis- and trans-4o $(20.3 \mathrm{mg}, 0.056$ mmol, 24\%; contaminated with 8 ) as a brown-yellow oil and a mixture of isoxazolidine trans-4o and regioisomers $7 \mathbf{o}(35.6 \mathrm{mg}, 0.098 \mathrm{mmol}$, $41 \%$ ) as a brown oil, after column chromatography (heptane $/ \mathrm{CH}_{2} \mathrm{Cl}_{2}$, 11:9). FTIR $\left[\bar{\nu}\left(\mathrm{cm}^{-1}\right)\right]$ of isoxazolidines cis- and trans-4o: 3413,3060 , 2962, 1599, 1436, 1258, 1110, 1022, 788, 756, 684, 660. FTIR $[\bar{\nu}$ $\left.\left(\mathrm{cm}^{-1}\right)\right]$ of isoxazolidine trans-4o and regioisomers $7 \mathbf{0}: 3406,3059$, 2963, 1597, 1455, 1231, 1107, 1014, 793, 693. Total yield: 65\%.

rac-(3R,5R)-3-(1H-Indol-2-yl)-2-phenyl-5-[(trifluoromethyl)sulfanyl]isoxazolidine (cis-4o). ${ }^{1} \mathrm{H}$ NMR $[500 \mathrm{MHz}, \delta$ (ppm), $\left.\mathrm{CDCl}_{3}\right]: 8.67(\mathrm{bs}, 1 \mathrm{H}), 7.61-7.58(\mathrm{~m}, 1 \mathrm{H}), 7.40-7.37(\mathrm{~m}, 1 \mathrm{H})$, 7.25-7.18 (m, $3 \mathrm{H}), 7.15-7.12(\mathrm{~m}, 1 \mathrm{H}), 7.05-7.02(\mathrm{~m}, 3 \mathrm{H}), 6.50(\mathrm{~d}$, $J=2.0 \mathrm{~Hz}, 1 \mathrm{H}), 6.07(\mathrm{dd}, J=8.3,3.5 \mathrm{~Hz}, 1 \mathrm{H}), 4.77(\mathrm{dd}, J=9.4,5.6$ $\mathrm{Hz}, 1 \mathrm{H}), 3.44-3.32(\mathrm{~m}, 1 \mathrm{H}), 2.50$ (ddd, $J=13.8,5.6,3.5 \mathrm{~Hz}, 1 \mathrm{H})$. ${ }^{13} \mathrm{C}$ NMR $\left[126 \mathrm{MHz}, \delta(\mathrm{ppm}), \mathrm{CDCl}_{3}\right]: 148.6,136.2,135.6,130.0$ (q, $J$ $=306.8 \mathrm{~Hz}), 129.1,128.6,123.9,122.6,120.6,120.4,116.7,111.5$, $100.9,80.7(q, J=2.4 \mathrm{~Hz}), 62.2,44.9 . R_{f}: 0.51$ (heptane $/ \mathrm{CH}_{2} \mathrm{Cl}_{2}, 1: 1$ ).

rac-(3R,5S)-3-(1H-Indol-2-yl)-2-phenyl-5-[(trifluoromethyl)sulfanyl]isoxazolidine (trans-4o). ${ }^{1} \mathrm{H}$ NMR $[500 \mathrm{MHz}, \delta(\mathrm{ppm})$, $\left.\mathrm{CDCl}_{3}\right]$ : 8.50 (bs, $\left.1 \mathrm{H}\right), 7.57-7.47(\mathrm{~m}, 3 \mathrm{H}), 7.32(\mathrm{dt}, J=8.2,0.9 \mathrm{~Hz}, 1$ $\mathrm{H}), 7.06-7.03(\mathrm{~m}, 1 \mathrm{H}), 6.98-6.90(\mathrm{~m}, 2 \mathrm{H}), 6.86-6.82(\mathrm{~m}, 2 \mathrm{H}), 6.40$ $(\mathrm{d}, J=2.1 \mathrm{~Hz}, 1 \mathrm{H}), 5.84(\mathrm{dd}, J=8.3,4.0 \mathrm{~Hz}, 1 \mathrm{H}), 4.28(\mathrm{dd}, J=9.4,5.7$ $\mathrm{Hz}, 1 \mathrm{H}), 2.82(\mathrm{dt}, J=13.7,8.9 \mathrm{~Hz}, 1 \mathrm{H}), 2.16-2.09(\mathrm{~m}, 1 \mathrm{H}) .{ }^{13} \mathrm{C}$ NMR [126 MHz, $\delta$ (ppm), $\left.\mathrm{CDCl}_{3}\right]: 148.5,134.4,132.7,129.0,128.6$, $122.6,122.5,120.6,119.5,116.2,111.3,100.5,82.1(\mathrm{q}, J=2.4 \mathrm{~Hz})$, 59.8, 44.6. $R_{f}: 0.42$ (heptane $/ \mathrm{CH}_{2} \mathrm{Cl}_{2}, 1: 1$ ).

3-(1H-Indol-2-yl)-2-phenyl-4-[(trifluoromethyl) sulfanyl]isoxazolidine (7o). ${ }^{1} \mathrm{H}$ NMR [500 MHz, $\delta(\mathrm{ppm}), \mathrm{CDCl}_{3}$ ]: 7.64-7.59 $(\mathrm{m}, 2 \mathrm{H}), 7.38-6.97(\mathrm{~m}, 16 \mathrm{H}), 6.59-6.55(\mathrm{~m}, 2 \mathrm{H}), 5.25(\mathrm{~d}, J=8.2$ $\mathrm{Hz}, 1 \mathrm{H}), 4.94(\mathrm{~d}, J=4.4 \mathrm{~Hz}, 1 \mathrm{H}), 4.67-4.61(\mathrm{~m}, 1 \mathrm{H}), 4.61-4.56(\mathrm{~m}$, $1 \mathrm{H}), 4.31(\mathrm{q}, J=7.6 \mathrm{~Hz}, 1 \mathrm{H}), 4.25-4.18(\mathrm{~m}, 2 \mathrm{H}), 4.11(\mathrm{dd}, J=9.3$, $6.2 \mathrm{~Hz}, 1 \mathrm{H}) .{ }^{13} \mathrm{C}$ NMR $\left[126 \mathrm{MHz}, \delta(\mathrm{ppm}), \mathrm{CDCl}_{3}\right]: 149.3,148.9$, $135.9,135.7,135.5,132.8,129.2,129.2,128.5,127.9,123.4,123.2$, $122.7,122.5,120.8,120.6,120.3,115.1,115.0,111.3,111.2,103.1$, $100.6,73.2,72.7,70.1,65.6,52.4,48.1$.

2-Phenyl-3-(1H-pyrrol-2-yl)-5-[(trifluoromethyl)sulfanyl]isoxazolidine (4p). According to the general procedure, the reaction of nitrone $6 \mathrm{p}$ (44 mg, $0.234 \mathrm{mmol}$ ) and alkene 2 in a $1.5 \mathrm{~mL}$ PTFE highpressure tube afforded a mixture of isoxazolidines cis- and trans- $4 \mathrm{p}$ and $7 \mathbf{p}$ (33 mg, $0.105 \mathrm{mmol}, 45 \%$ ) as a brown oil, after column chromatography (heptane/ $\mathrm{CH}_{2} \mathrm{Cl}_{2}, 4: 1 \rightarrow \mathrm{CH}_{2} \mathrm{Cl}_{2} / \mathrm{MeOH}, 10: 1$ ). FTIR $\left[\bar{\nu}\left(\mathrm{cm}^{-1}\right)\right]: 3421,2960,1597,1489,1154,1109,1082,1029,756$, 722. HRMS (EI) $\mathrm{m} / z:\left[\mathrm{M}^{\bullet}\right]$ calcd for $\mathrm{C}_{14} \mathrm{H}_{13} \mathrm{~F}_{3} \mathrm{~N}_{2} \mathrm{OS} 314.0701$, found 314.0706. $R_{f}$ (unassigned mixture): 0.46, 0.30, 0.24, 0.20 (heptane/ $\left.\mathrm{CH}_{2} \mathrm{Cl}_{2}, 1: 1\right)$. Total yield: $45 \%$.

rac-(3R,5R)-2-Phenyl-3-(1H-pyrrol-2-yl)-5-[(trifluoromethyl)sulfanyl]isoxazolidine (cis-4p). ${ }^{1} \mathrm{H}$ NMR $[400 \mathrm{MHz}, \delta$ (ppm), $\mathrm{CDCl}_{3}$ ]: 8.65 (bs, $\left.1 \mathrm{H}\right), 7.28-7.22(\mathrm{~m}, 2 \mathrm{H}), 7.08-6.98(\mathrm{~m}, 3 \mathrm{H})$, 6.81-6.77 (m, 1 H), 6.21-6.18 (m, 1 H), 6.15-6.12 (m, 1 H), 6.03 $(\mathrm{dd}, J=8.3,3.6 \mathrm{~Hz}, 1 \mathrm{H}), 4.62(\mathrm{dd}, J=8.9,5.6 \mathrm{~Hz}, 1 \mathrm{H}), 3.34-3.17(\mathrm{~m}$, $1 \mathrm{H}), 2.48-2.36(\mathrm{~m}, 1 \mathrm{H}) .{ }^{13} \mathrm{C} \mathrm{NMR}\left[101 \mathrm{MHz}, \delta(\mathrm{ppm}), \mathrm{CDCl}_{3}\right]: \delta$ $148.9,130.0(q, J=307.9 \mathrm{~Hz}), 129.0,128.8,123.8,116.8,115.2,109.1$, 106.7, $80.7(\mathrm{q}, J=2.3 \mathrm{~Hz}), 62.5$, 44.6 .

rac-(3R,5S)-2-Phenyl-3-(1H-pyrrol-2-yl)-5-[(trifluoromethyl)sulfanyl]isoxazolidine (trans-4p). ${ }^{1} \mathrm{H}$ NMR $[500 \mathrm{MHz}, \delta(\mathrm{ppm})$, $\mathrm{CDCl}_{3}$ ]: 8.57 (bs, $\left.1 \mathrm{H}\right), 7.31-6.66(\mathrm{~m}, 7 \mathrm{H}), 6.27-6.15(\mathrm{~m}, 1 \mathrm{H}), 5.97$ $(\mathrm{t}, J=6.4 \mathrm{~Hz}, 1 \mathrm{H}), 5.07(\mathrm{dd}, J=7.0,4.4 \mathrm{~Hz}, 1 \mathrm{H}), 3.00(\mathrm{ddd}, J=12.2$, $7.1,4.4 \mathrm{~Hz}, 1 \mathrm{H}), 2.68(\mathrm{dt}, J=13.1,6.4 \mathrm{~Hz}, 1 \mathrm{H}) .{ }^{13} \mathrm{C}$ NMR $[101 \mathrm{MHz}$, $\left.\delta(\mathrm{ppm}), \mathrm{CDCl}_{3}\right]$ : 150.0, 129.9, 128.9, 123.8, 118.0, 115.8, 107.2, 106.3, 82.1, 62.7, 43.2. The carbon signal of $\mathrm{SCF}_{3}$ was not observed.

2-Phenyl-3-(1 H-pyrrol-2-yl)-4-[(trifluoromethyl)sulfanyl]isoxazolidine (7p). ${ }^{1} \mathrm{H}$ NMR [400 MHz, $\delta$ (ppm), $\left.\mathrm{CDCl}_{3}\right]: 8.65$ (bs, 1 $\mathrm{H}), 7.30-7.23(\mathrm{~m}, 2 \mathrm{H}), 7.06-6.99(\mathrm{~m}, 3 \mathrm{H}), 6.81-6.77(\mathrm{~m}, 1 \mathrm{H})$, $6.27-6.19(\mathrm{~m}, 1 \mathrm{H}), 6.19(\mathrm{~m}, 1 \mathrm{H}), 5.11(\mathrm{~d}, J=7.8 \mathrm{~Hz}, 1 \mathrm{H}), 4.66-4.55$ $(\mathrm{m}, 1 \mathrm{H}), 4.22(\mathrm{q}, J=7.5 \mathrm{~Hz}, 1 \mathrm{H}), 4.15(\mathrm{dd}, J=8.6,6.8 \mathrm{~Hz}, 1 \mathrm{H}) .{ }^{13} \mathrm{C}$ NMR [101 MHz, $\delta$ (ppm), $\left.\mathrm{CDCl}_{3}\right]: 149.2,129.3,126.0,123.4,118.8$,
115.2, 109.0, 108.8, 73.5, 65.3, $48.7(\mathrm{q}, J=1.3 \mathrm{~Hz})$. The carbon signal of $\mathrm{SCF}_{3}$ was not observed.

2-Phenyl-3-(1H-pyrrol-2-yl)-4-[(trifluoromethyl)sulfanyl]isoxazolidine (7p). ${ }^{1} \mathrm{H}$ NMR [500 MHz, $\left.\delta(\mathrm{ppm}), \mathrm{CDCl}_{3}\right]$ : 8.57 (bs, 1 $\mathrm{H}), 7.31-6.66(\mathrm{~m}, 7 \mathrm{H}), 6.27-6.15(\mathrm{~m}, 1 \mathrm{H}), 4.82(\mathrm{~d}, J=4.2 \mathrm{~Hz}, 1 \mathrm{H})$, 4.65-4.50 (m, $2 \mathrm{H}), 4.26-4.10(\mathrm{~m}, 1 \mathrm{H}) .{ }^{13} \mathrm{C}$ NMR $[101 \mathrm{MHz}, \delta$ (ppm), $\left.\mathrm{CDCl}_{3}\right]: 149.6,129.4,126.0,124.1,118.1,115.2,109.1,108.7$, 70.3, 65.3, 48.7. The carbon signal of $\mathrm{SCF}_{3}$ was not observed.

3-(Furan-2-yl)-2-phenyl-5-[(trifluoromethyl)sulfanyl]isoxazolidine $(4 q)$. According to the general procedure, the reaction of nitrone $\mathbf{6 q}$ (44 mg, $0.234 \mathrm{mmol}$ ) in a $1.5 \mathrm{~mL}$ PTFE high-pressure tube afforded isoxazolidine cis-4q $(33.2 \mathrm{mg}, 0.105 \mathrm{mmol}, 45 \%)$ as an off white solid and isoxazolidines trans- $\mathbf{4 q}$ and $7 \mathbf{q}(24.7 \mathrm{mg}, 0.078 \mathrm{mmol}, 34 \%)$ as a yellow oil, after column chromatography (heptane $/ \mathrm{CH}_{2} \mathrm{Cl}_{2}, 23: 2 \rightarrow$ heptane $\left./ \mathrm{CH}_{2} \mathrm{Cl}_{2}, 0: 1\right)$. Total yield: $79 \%$.

rac-(3R,5R)-3-(Furan-2-yl)-2-phenyl-5-[(trifluoromethyl)sulfanyl]isoxazolidine (cis-4q). ${ }^{1} \mathrm{H}$ NMR $\left[400 \mathrm{MHz}, \delta(\mathrm{ppm}), \mathrm{CDCl}_{3}\right]$ : 7.45 (dd, $J=1.8,0.9 \mathrm{~Hz}, 1 \mathrm{H}), 7.31-7.22(\mathrm{~m}, 2 \mathrm{H}), 7.10-6.98(\mathrm{~m}, 3 \mathrm{H})$, $6.37(\mathrm{dd}, J=3.2,1.8 \mathrm{~Hz}, 1 \mathrm{H}), 6.35(\mathrm{~d}, J=3.2 \mathrm{~Hz}, 1 \mathrm{H}), 6.00(\mathrm{dd}, J=$ $8.1,4.5 \mathrm{~Hz}, 1 \mathrm{H}), 4.50(\mathrm{dd}, J=8.8,6.5 \mathrm{~Hz}, 1 \mathrm{H}), 3.23$ (dddq, $J=13.5$, 8.8, 8.1, $0.9 \mathrm{~Hz}, 1 \mathrm{H}), 2.63$ (ddd, $J=13.5,6.5,4.5 \mathrm{~Hz}, 1 \mathrm{H}) .{ }^{13} \mathrm{C}$ NMR $\left[101 \mathrm{MHz}, \delta(\mathrm{ppm}), \mathrm{CDCl}_{3}\right]: 151.0,148.7,143.0,130.2(\mathrm{q}, J=307.7$ $\mathrm{Hz}), 128.9,124.2,117.5,110.8,109.0,80.5(\mathrm{q}, J=2.6 \mathrm{~Hz}), 63.0,42.4$ $(\mathrm{q}, J=1.2 \mathrm{~Hz})$. FTIR $\left[\bar{\nu}\left(\mathrm{cm}^{-1}\right)\right]: 2922,1489,1453,1254,1106,1037$, 936, 743, 680. HRMS (EI) $\mathrm{m} / z$ : $\left[\mathrm{M}^{+\bullet}\right]$ calcd for $\mathrm{C}_{14} \mathrm{H}_{12} \mathrm{~F}_{3} \mathrm{NO}_{2} \mathrm{~S}$ 315.0541, found 315.0538. $R_{f}: 0.48$ (heptane $/ \mathrm{CH}_{2} \mathrm{Cl}_{2}, 7: 3$ ).

rac-(3R,5S)-3-(Furan-2-yl)-2-phenyl-5-[(trifluoromethyl)sulfanyl]isoxazolidine (trans-4q). ${ }^{1} \mathrm{H}$ NMR $\left[400 \mathrm{MHz}, \delta\right.$ (ppm), $\left.\mathrm{CDCl}_{3}\right]: 7.37$ (dd, $J=1.8,0.9 \mathrm{~Hz}, 1 \mathrm{H}), 7.27-7.22(\mathrm{~m}, 2 \mathrm{H}), 7.09-7.05(\mathrm{~m}, 2 \mathrm{H})$, $6.98(\mathrm{tt}, J=7.3,1.2 \mathrm{~Hz}, 1 \mathrm{H}), 6.30(\mathrm{dd}, J=3.3,1.8 \mathrm{~Hz}, 1 \mathrm{H}), 6.26(\mathrm{dt}, J$ $=3.3,0.8,1 \mathrm{H}), 6.11(\mathrm{dd}, J=7.4,5.2 \mathrm{~Hz}, 1 \mathrm{H}), 5.00(\mathrm{dd}, J=7.7,4.3,1$ $\mathrm{H}), 3.17(\mathrm{dddq} J=13.7,7.4,4.3,0.8,1 \mathrm{H}), 2.77-2.67(\mathrm{~m}, 1 \mathrm{H}) .{ }^{13} \mathrm{C}$ NMR [101 MHz, $\delta$ (ppm), $\left.\mathrm{CDCl}_{3}\right]: 151.6,149.2,142.8,130.0$ (q, $J=$ $307.7 \mathrm{~Hz}), 128.8,123.0,116.2,110.6,108.4,81.8(\mathrm{q}, J=2.5 \mathrm{~Hz}), 61.6$, $41.6(\mathrm{q}, J=1.2 \mathrm{~Hz})$. FTIR $\left[\bar{\nu}\left(\mathrm{cm}^{-1}\right)\right]: 2872,1599,1490,1262,1118$, 1046, 757, 694. HRMS (EI) $\mathrm{m} / z$ : $\left[\mathrm{M}^{+\bullet}\right]$ calcd for $\mathrm{C}_{14} \mathrm{H}_{12} \mathrm{~F}_{3} \mathrm{NO}_{2} \mathrm{~S}$ 315.0541, found 315.0545. $R_{f}: 0.39$ (heptane $/ \mathrm{CH}_{2} \mathrm{Cl}_{2}, 7: 3$ ).

N-Benzyl-2-phenyl-5-[(trifluoromethyl)sulfanyl]isoxazolidine-3carboxamide (4r). According to the general procedure, the reaction of nitrone $6 \mathrm{r}(25 \mathrm{mg}, 0.098 \mathrm{mmol})$ and alkene 2 in a $1 \mathrm{~mL}$ PTFE highpressure tube afforded isoxazolidine cis-4r $(3.2 \mathrm{mg}, 0.0084 \mathrm{mmol}, 9 \%$; used for NMR characterization) as a white solid and a mixture of isoxazolidines cis- and trans- $4 \mathbf{r}(16.1 \mathrm{mg}, 0.042 \mathrm{mmol}, 51 \%)$ as a yellowwhite solid, after column chromatography (heptane $/ \mathrm{CH}_{2} \mathrm{Cl}_{2}, 17: 3 \rightarrow$ $\left.\mathrm{CH}_{2} \mathrm{Cl}_{2} / \mathrm{MeOH}, 24: 1\right)$. FTIR $\left[\bar{\nu}\left(\mathrm{cm}^{-1}\right)\right]: 3385,33092930,1668$, 1597, 1454, 1161, 1029, 697. HRMS (EI) $\mathrm{m} / z:\left[\mathrm{M}^{+\bullet}\right]$ calcd for $\mathrm{C}_{18} \mathrm{H}_{17} \mathrm{~F}_{3} \mathrm{~N}_{2} \mathrm{O}_{2} \mathrm{~S} 382.0963$, found 382.0964 . Total yield: $60 \%$.

rac-(3R,5R)-N-Benzyl-2-phenyl-5-[(trifluoromethyl)sulfanyl]isoxazolidine-3-carboxamide (cis-4r). ${ }^{1} \mathrm{H}$ NMR [400 MHz, $\delta(\mathrm{ppm})$, $\mathrm{CDCl}_{3}$ ]: 7.59-7.52 (m, $\left.1 \mathrm{H}\right), 7.37-7.27(\mathrm{~m}, 7 \mathrm{H}), 7.11-7.03(\mathrm{~m}, 1$ $\mathrm{H}), 7.02-6.97(\mathrm{~m}, 2 \mathrm{H}), 5.88(\mathrm{dd}, J=8.1,4.7 \mathrm{~Hz}, 1 \mathrm{H}), 4.56(\mathrm{dd}, J=$ $14.8,6.1 \mathrm{~Hz}, 1 \mathrm{H}), 4.49(\mathrm{dd}, J=14.8,5.9 \mathrm{~Hz}, 1 \mathrm{H}), 4.31(\mathrm{dd}, J=9.6,3.5$ $\mathrm{Hz}, 1 \mathrm{H}), 3.19-3.07(\mathrm{~m}, 1 \mathrm{H}), 2.75-2.65(\mathrm{~m}, 1 \mathrm{H}) .{ }^{13} \mathrm{C}$ NMR [101 $\left.\mathrm{MHz}, \delta(\mathrm{ppm}), \mathrm{CDCl}_{3}\right]: 169.7,148.8,137.6,129.6(\mathrm{q}, J=308.1 \mathrm{~Hz})$, $129.5,128.9,127.9,127.8,123.9,115.1,80.3(\mathrm{q}, J=2.5 \mathrm{~Hz}), 67.6,43.7$, 39.4 .

rac-(3R,5S)-N-Benzyl-2-phenyl-5-[(trifluoromethyl)sulfanyl]isoxazolidine-3-carboxamide (trans-4r). ${ }^{1} \mathrm{H}$ NMR $[500 \mathrm{MHz}, \delta$ (ppm), $\mathrm{CDCl}_{3}$ ]: 7.52-7.43 (m, 1 H), 7.36-7.23 (m, $\left.7 \mathrm{H}\right), 7.11-7.02$ $(\mathrm{m}, 3 \mathrm{H}), 5.91(\mathrm{t}, J=6.8 \mathrm{~Hz}, 1 \mathrm{H}), 4.54-4.49(\mathrm{~m}, 2 \mathrm{H}), 4.46(\mathrm{dd}, J=$ $15.0,5.6 \mathrm{~Hz}, 1 \mathrm{H}), 3.29$ (ddd, $J=13.5,7.3,3.9 \mathrm{~Hz}, 1 \mathrm{H}), 2.62$ (ddd, $J=$ $13.9,8.1,6.2 \mathrm{~Hz}, 1 \mathrm{H}) .{ }^{13} \mathrm{C}$ NMR [126 MHz, $\left.\delta(\mathrm{ppm}), \mathrm{CDCl}_{3}\right]: 169.1$, $149.4,137.6,129.6(q, J=308.1 \mathrm{~Hz}), 129.0,128.8,127.7,127.6,123.6$, $115.1,82.3(\mathrm{q}, J=2.5 \mathrm{~Hz}), 67.1,43.5,39.0$.

\section{ASSOCIATED CONTENT}

\section{Supporting Information}

The Supporting Information is available free of charge on the ACS Publications website at DOI: 10.1021/acs.joc.7b02639. 
Computational details, ASA and EDA of $6 \mathrm{~m}$ and $6 \mathrm{p}$, and Cartesian coordinates (PDF)

Detailed optimization reaction conditions; ${ }^{1} \mathrm{H}$ and ${ }^{13} \mathrm{C}$ NMR spectra (PDF)

\section{AUTHOR INFORMATION}

\section{Corresponding Authors}

*E-mail: f.m.bickelhaupt@vu.nl.

*E-mail: floris.rutjes@ru.nl.

\section{ORCID}

Trevor A. Hamlin: 0000-0002-5128-1004

F. Matthias Bickelhaupt: 0000-0003-4655-7747

Floris P. J. T. Rutjes: 0000-0003-1538-3852

Notes

The authors declare no competing financial interest.

\section{ACKNOWLEDGMENTS}

This work has been supported by the FP7Marie Curie Actions of the European Commission via the ITN ECHONET Network (MCITN-2012-316379) and NWO via the Planetary and Exoplanetary Science program (PEPSci) and the Dutch Astrochemistry Network (DAN). We thank Dr. Paul B. White for his help in the assignment of the diastereo- and regioisomers, and Birgit Kuhn (Bayer AG) for the helpful discussions.

\section{REFERENCES}

(1) (a) Xu, X. H.; Matsuzaki, K.; Shibata, N. Chem. Rev. 2015, 115, 731-764. (b) Liu, J.; Chu, L.; Qing, F. L. Org. Lett. 2013, 15, 894-897. (c) Yang, Y. D.; Azuma, A.; Tokunaga, E.; Yamasaki, M.; Shiro, M.; Shibata, N. J. Am. Chem. Soc. 2013, 135, 8782-8785. (d) Zhang, C. P.; Vicic, D. A. J. Am. Chem. Soc. 2012, 134, 183-185. (e) Hansch, C.; Leo, A.; Unger, S. H.; Kim, K. H.; Nikaitani, D.; Lien, E. J. J. Med. Chem. 1973, 16, 1207-1216.

(2) Aswapokee, N.; Neu, H. C. Antimicrob. Agents Chemother. 1979, 15, 444-446.

(3) Pommier, P.; Keita, A.; Wessel-Robert, S.; Dellac, B.; Mundt, H. C. Rev. Med. Vet. 2003, 154, 41.

(4) Yagupolskii, L. M.; Maletina, I. I.; Petko, K. I.; Fedyuk, D. V.; Handrock, R.; Shavaran, S. S.; Klebanov, B. M.; Herzig, S. J. Fluorine Chem. 2001, 109, 87-94.

(5) (a) Jiang, L.; Qian, J.; Yi, W.; Lu, G.; Cai, C.; Zhang, W. Angew. Chem., Int. Ed. 2015, 54, 14965-14969. (b) Shao, X.; Wang, X.; Yang, T.; Lu, L.; Shen, Q. Angew. Chem., Int. Ed. 2013, 52, 3457-3460. (c) Weng, Z.; He, W.; Chen, C.; Lee, R.; Tan, D.; Lai, Z.; Kong, D.; Yuan, Y.; Huang, K. W. Angew. Chem., Int. Ed. 2013, 52, 1548-1552. (d) Yang, Y. D.; Azuma, A.; Tokunaga, E.; Yamasaki, M.; Shiro, M.; Shibata, N. J. Am. Chem. Soc. 2013, 135, 8782-8785. (e) Zhang, C. P.; Vicic, D. A. J. Am. Chem. Soc. 2012, 134, 183-185. (f) Teverovskiy, G.; Surry, D. S.; Buchwald, S. L. Angew. Chem., Int. Ed. 2011, 50, 73127314.

(6) (a) Kieltsch, I.; Eisenberger, P.; Togni, A. Angew. Chem., Int. Ed. 2007, 46, 754-757. (b) Pooput, C.; Dolbier, W. R., Jr.; Médebielle, M. J. Org. Chem. 2006, 71, 3564-3568. (c) Pooput, C.; Médebielle, M.; Dolbier, W. R., Jr Org. Lett. 2004, 6, 301-303. (d) Billard, T.; Large, S.; Langlois, B. R. Tetrahedron Lett. 1997, 38, 65-68.

(7) Harris, J. F., Jr J. Org. Chem. 1967, 32, 2063-2074.

(8) Haas, A.; Hinsken, W. J. Fluorine Chem. 1985, 28, 303-317.

(9) For examples of 1,3-dipolar cycloaddition reactions between alkenes and nitrones, see: (a) Chakraborty, B.; Luitel, G. P. Tetrahedron Lett. 2013, 54, 765-770. (b) Gothelf, K. V.; Jørgensen, K. A. Chem. Commun. 2000, 1449-1458. (c) Simonsen, K. B.; Bayón, P.; Hazell, R. G.; Gothelf, K. V.; Jørgensen, K. A. J. Am. Chem. Soc. 1999, 121, 38453853.

(10) (a) Blanco-Ania, D.; Aben, R. W. M.; van Berkom, L. W. A.; Scheeren, H. W.; Rutjes, F. P. J. T. Eur. J. Org. Chem. 2017, 2017,
3729-3737. (b) Blanco-Ania, D.; Aben, R. W. M.; van Berkom, L. W. A.; Scheeren, H. W.; Rutjes, F. P. J. T. Eur. J. Org. Chem. 2014, 2014, 1438-1444. (c) Matsumoto, K.; Hamana, H.; Iida, H. Helv. Chim. Acta 2005, 88, 2033-2234. (d) Jenner, G. Tetrahedron 2002, 58, 51855202.

(11) Becker, A. R.; Sternson, L. A. Proc. Natl. Acad. Sci. U. S. A. 1981, 78, 2003-2007.

(12) Penoni, A.; Volkmann, J.; Nicholas, K. M. Org. Lett. 2002, 4, 699-701.

(13) It is important to note that the assignment of the cis- and transdiastereoisomers of 7 could not be clarified by NMR studies.

(14) (a) Becke, A. D. Phys. Rev. A: At., Mol., Opt. Phys. 1988, 38, 3098-3100. (b) Perdew, J. P. Phys. Rev. B: Condens. Matter Mater. Phys. 1986, 33, 8822-8824. (c) Van Lenthe, E.; Baerends, E. J. J. Comput. Chem. 2003, 24, 1142-1156.

(15) (a) te Velde, G.; Bickelhaupt, F. M.; Baerends, E. J.; Fonseca Guerra, C.; van Gisbergen, S. J. A.; Snijders, J. G.; Ziegler, T. J. Comput. Chem. 2001, 22, 931-967. (b) Fonseca Guerra, C.; Snijders, J. G.; te Velde, G.; Baerends, E. J. Theor. Chem. Acc. 1998, 99, 391-403.

(16) (a) Pye, C. C.; Ziegler, T. Theor. Chem. Acc. 1999, 101, 396-408. (b) Klamt, A.; Schüürmann, G. J. Chem. Soc., Perkin Trans. 2 1993, 2 799-805. (c) Klamt, A. J. Phys. Chem. 1995, 99, 2224-2235. (d) Klamt, A.; Jonas, V. J. Chem. Phys. 1996, 105, 9972-9981.

(17) (a) Bickelhaupt, F. M.; Houk, K. N. Angew. Chem., Int. Ed. 2017, 56, 10070-10086. (b) Wolters, L. P.; Bickelhaupt, F. M. WIRES Comput. Mol. Sci. 2015, 5, 324-343. (c) Bickelhaupt, F. M. J. Comput. Chem. 1999, 20, 114-128.

(18) (a) Ess, D. H.; Houk, K. N. J. Am. Chem. Soc. 2008, 130, 1018710198. (b) Ess, D. H.; Houk, K. N. J. Am. Chem. Soc. 2007, 129, 10646-10647.

(19) Bickelhaupt, F. M.; Baerends, E. J. In Reviews in Computational Chemistry; Lipkowitz, K. B., Boyd, D. B., Eds.; VCH: New York, 2000; Vol. 15, pp 1-86.

(20) Ung, S.; Falguières, A.; Guy, A.; Ferroud, C. Tetrahedron Lett. 2005, 46, 5913-5917.

(21) Wheeler, O. H.; Gore, P. H. J. Am. Chem. Soc. 1956, 78, $3363-$ 3366.

(22) Raunak; Kumar, V.; Mukherjee, S.; Poonam; Prasad, A. K.; Olsen, C. E.; Schaeffer, S. J. C.; Sharma, S. K.; Watterson, A. C.; Errington, W.; Parmar, V. S. Tetrahedron 2005, 61, 5687-5697.

(23) Jiao, P.; Nakashima, D.; Yamamoto, H. Angew. Chem., Int. Ed. 2008, 47, 2411-2413.

(24) Ito, S.; Kubota, Y.; Asami, M. Tetrahedron Lett. 2014, 55, 49304932.

(25) Xie, L.; Bai, H.; Li, J.; Yu, X.; Zhang, Z.; Fu, B. Tetrahedron 2017, 73, 2923-2930.

(26) Poulsen, P. H.; Vergura, S.; Monleón, A.; Jørgensen, D. K. B.; Jørgensen, K. A. J. Am. Chem. Soc. 2016, 138, 6412-6415.

(27) Dooley, B. M.; Bowles, S. E.; Storr, T.; Frank, N. L. Org. Lett. 2007, 9, 4781-4783.

(28) Andrade, M. M.; Barros, M. T.; Pinto, R. C. Tetrahedron 2008, 64, 10521-10530. 